\title{
Supersymmetry breaking, brane dynamics and Swampland conjectures
}

\author{
Ivano Basile \\ Service de Physique de l'Univers, Champs et Gravitation, Université de Mons, \\ Place du Parc 20, 7000 Mons, Belgium \\ E-mail: ivano.basile@umons.ac.be
}

ABSTRACT: We investigate interactions between branes of various dimensions, both charged and uncharged, in three non-supersymmetric string models. These include the USp(32) and $\mathrm{U}(32)$ orientifold projections of the type IIB and type 0B strings, as well as the $\mathrm{SO}(16) \times \mathrm{SO}(16)$ projection of the exceptional heterotic string. The resulting ten-dimensional spectra are free of tachyons, and the combinations of branes that they contain give rise to rich and varied dynamics. We compute static potentials for parallel stacks of branes in three complementary regimes: the probe regime, in which one of the two stacks is parametrically heavier than the other, the string-amplitude regime, in which both stacks are light, and the holographic regime. Whenever comparisons are possible, we find qualitative agreement despite the absence of supersymmetry. For charged branes, our analysis reveals that the Weak Gravity Conjecture is satisfied in a novel way via a renormalization of the effective charge-to-tension ratio.

KEYwords: D-branes, $p$-branes, Supersymmetry Breaking, AdS-CFT Correspondence

ArXiv EPrint: 2106.04574 


\section{Contents}

1 Introduction 1

2 Branes and gravitational tadpoles $\quad 3$

2.1 Charged and uncharged branes 4

2.2 Low-energy effective description 4

3 Back-reaction of non-supersymmetric branes $\quad 6$

3.1 Back-reaction of extremal branes 6

3.1.1 Nucleation in AdS and brane tension $\quad 7$

3.2 Static Dudas-Mourad solutions as 8-branes 9

$\begin{array}{lll}3.3 & \text { D3-branes in the type } 0^{\prime} \mathrm{B} \text { model } & 10\end{array}$

4 Brane interactions and the WGC 11

4.1 Probe potentials and Weak Gravity 12

$\begin{array}{lll}\text { 4.1.1 Repulsive forces between extremal branes } & 13\end{array}$

$\begin{array}{lll}\text { 4.1.2 } & \text { Brane probes in the Dudas-Mourad geometry } & 17\end{array}$

4.1.3 Probe 8-branes in AdS $\times \mathbb{S}$ throats 19

$\begin{array}{lll}4.2 & \text { String-amplitude regime } & 21\end{array}$

4.3 Holographic regime 22

\section{Introduction}

Achieving a deeper understanding of string theory, and quantum gravity in general, as well as building stronger ties with phenomenology, requires addressing the problem of supersymmetry breaking. Despite remarkable progress has been accomplished in a variety of directions, the lack of an overarching guiding principle together with numerous issues in low-energy effective field theory (EFT) constructions points to high-energy supersymmetry breaking as the natural environment to seek instructive lessons, both from a theoretical and a phenomenological perspective. Indeed, it is possible to concoct perturbative models in which supersymmetry is either absent or broken at the string scale. Within the scope of this paper, the former setting comprise the $\mathrm{SO}(16) \times \mathrm{SO}(16)$ heterotic model of $[1,2]$ and the U(32) "type $0^{\prime} \mathrm{B}$ " model of $[3,4]$, while the latter setting is embodied by the USp(32) model of [5], which exhibits the peculiar phenomenon of "brane supersymmetry breaking" (BSB) [6-9]. ${ }^{1}$ The notion that high-energy supersymmetry breaking is in some sense natural also resonates with recent considerations stemming from the Swampland program [11, 12], and in particular the central role played by the gravitino mass is suggestively reminiscent of BSB, as discussed in [10].

\footnotetext{
${ }^{1}$ See also [10] for a recent investigation of models featuring a novel type of BSB.
} 
At any rate, in order to elucidate deeper features of supersymmetry breaking it appears paramount to go beyond the EFT regime. Specifically, one can consider the dynamics of branes, which manifest themselves in various guises in different regimes of string theory, ranging from soliton solutions of space-time field equations to conformal field theory (CFT) boundary states to world-volume gauge theories. They have been proven an invaluable tool to investigate any setting, supersymmetric or not, and in this paper we would like to pursue this approach to shed some light on the subtle, and so far elusive, physics of high-energy supersymmetry breaking. Concretely, we shall compute static interaction potentials between stacks of parallel branes of various charges and dimensions, comparing the results obtained in different regimes. In particular, the interactions between extremal branes reveal a peculiar, novel mechanism giving rise to a repulsive force [13], which we shall discuss in detail in the following, further grounding the connections between top-down settings and the Swampland program that were developed in [14].

The contents of this paper are organized as follows. In section 2 we provide an overview of the non-supersymmetric string models at stake, describing their brane content in section 2.1 and low-energy EFT description in section 2.2. In section 3 we discuss the gravitational back-reaction of branes of various dimensions, namely uncharged D8-branes and charged D1, D3 and NS5-branes. The latter feature near-horizon Anti-de Sitter (AdS) throats, characteristic of extremal black objects, with the exception of D3-branes in the type $0^{\prime} \mathrm{B}$ model of $[3,4]$ which feature quasi-AdS geometries that converge non-uniformly to $\mathrm{AdS}_{5} \times \mathbb{S}^{5}$ for a large number of branes $[15,16]$. In section 4 we present in detail the computation of interaction potentials for parallel brane stacks of various dimensions and charges. Our analysis spans three complementary regimes, depending on the numbers $N_{1}, N_{2}$ of branes in the two stacks. In particular, for $N_{1} \ll N_{2}$ the branes in the first stack effectively probe the space-time geometry sourced by the second stack, while for $N_{1}, N_{2}=\mathcal{O}(1)$ the interaction potential is calculable via perturbative string amplitudes, at least in principle. Whenever both stacks have the same dimensions and charges the resulting expressions are highly involved, but in all other cases the leading contribution for large brane separations can be extracted from the annulus amplitude. Finally, we study the world-volume gauge theory of D1-branes in the USp(32) model of [5], where the bosonic and fermionic contributions to the one-loop effective potential do not cancel, leading to a non-trivial result along the lines of $[17,18]$.

The emergence of an $\mathrm{AdS}_{3}$ throat for a large number of D1-branes suggests a holographic interpretation of the results, opening new avenues in top-down holography with broken supersymmetry. Moreover, branes with the same charges always repel, corroborating the Weak Gravity Conjecture (WGC) [19] in a non-supersymmetric context. In the probe regime, this behavior stems from a novel renormalization of the effective charge-to-tension ratio due to the supersymmetry-breaking gravitational tadpoles [13], while in the world-volume gauge theory it stems from the absence of the fermionic contribution to the one-loop effective potential. More generally, the qualitative repulsive or attractive behavior is shared among different regimes whenever they apply. This non-trivial agreement points to a tantalizing deeper principle behind our findings. 


\section{Branes and gravitational tadpoles}

In this section we briefly review the three non-supersymmetric string models that we shall investigate on in this paper, focusing on their brane content. In particular, the USp(32) model of [5] and the $\mathrm{U}(32)$ type $0^{\prime} \mathrm{B}$ model of $[3,4]$ contain several charged and uncharged branes in their perturbative spectra [20]. In suitable probe regimes, their dynamics can be studied via string amplitudes or effective world-volume actions, while in the opposite regime their back-reaction can be described from the bulk gravitational action. Similarly, the heterotic NS5-branes in the $\mathrm{SO}(16) \times \mathrm{SO}(16)$ model of $[1,2]$ can be studied at low energies, despite the absence of strong-weak dualities. ${ }^{2}$

While the type $0^{\prime} \mathrm{B}$ model arises as a non-tachyonic orientifold of the tachyonic type 0B model, and is thus non-supersymmetric at the outset, the USp(32) model and the $\mathrm{SO}(16) \times \mathrm{SO}(16)$ heterotic model arise as projections of the type IIB and the $E_{8} \times E_{8}$ superstrings respectively, thereby featuring supersymmetry breaking at the string scale.

The relevant orientifold projections [25-32] can be constructed via one-loop vacuum amplitudes, ${ }^{3}$ in a similar fashion to that of the type I superstring. In particular, one can obtain the USp(32) model of [5] introducing an O9-plane with positive tension and charge together with $\overline{\mathrm{D} 9}$-branes, yielding a vanishing R-R tadpole. However, the NS-NS tadpole is not canceled, and thus supersymmetry is broken at the string scale. ${ }^{4}$ Since the residual tension in the NS-NS tadpole does not cancel, the low-energy physics of this model includes the string-frame runaway exponential potential ${ }^{5}$

$$
T \int d^{10} x \sqrt{-g_{\mathrm{s}}} e^{-\phi}
$$

whose Einstein-frame counterpart is

$$
T \int d^{10} x \sqrt{-g} e^{\gamma \phi}, \quad \gamma=\frac{3}{2} .
$$

Similar exponential potentials actually appear also in the other models at stake, and resonate with the considerations in $[39,40]$. We shall review some aspects of their dramatic back-reaction in the next section. The $\mathrm{U}(32)$ type $0^{\prime} \mathrm{B}$ model of $[3,4]$ arises instead from a projection of the type 0B model. The corresponding O9-plane, which arises from a particular combination of elementary O9-planes [34], has vanishing tension, and therefore the relevant exponential potential is halved with respect to eq. (2.2).

Finally, the $E_{8} \times E_{8}$ superstring admits a tachyon-free projection that breaks supersymmetry $[1,2]$. In particular, one can project onto the states with even total fermion number, ${ }^{6}$

\footnotetext{
${ }^{2}$ See $[21,22]$ for some efforts in this direction. A possible non-perturbative construction of the $\operatorname{SO}(16) \times$ $\mathrm{SO}(16)$ heterotic model, along the lines of Hořava-Witten theory [23], has been proposed in [24]. It would be interesting to describe NS5-branes in this setting, and I would like to thank A. Faraggi for pointing this out.

${ }^{3}$ The following construction is based on the characters $\left(O_{2 n}, V_{2 n}, S_{2 n}, C_{2 n}\right)$ of the level-one affine $\mathfrak{s o}(2 n)$ algebra. See [33-36] for reviews.

${ }^{4}$ More precisely, supersymmetry is preserved in the closed-string sector, but it is non-linearly realized in the open-string sector via "brane supersymmetry breaking" (BSB) [6-9].

${ }^{5}$ See $[37,38]$ for more details on the low-energy couplings of BSB models.

${ }^{6}$ Let us recall that $\mathrm{T}$-duality corresponds to a projection onto the states with even right-moving fermion number.
} 
and taking modular invariance into account the final perturbative spectrum is devoid of tacyhons by virtue of level matching. However, the one-loop vacuum energy does not vanish, ${ }^{7}$ and its value is of order one in string units. In the string-frame low-energy effective action it appears as a cosmological constant, and thus as a runaway exponential potential

$$
T \int d^{10} x \sqrt{-g} e^{\gamma \phi}, \quad \gamma=\frac{5}{2}
$$

in the Einstein frame. All in all, the low-energy manifestation of gravitational tadpoles in both the orientifold models and in the $\mathrm{SO}(16) \times \mathrm{SO}(16)$ heterotic model can be encompassed by the same type of exponential potential for the dilaton.

\subsection{Charged and uncharged branes}

One-loop vacuum amplitudes can be also employed to investigate the D-brane content of the orientifold models, as described in detail in [20]. Indeed, the consistency of (transversechannel) $\mathrm{D} p$ - $\mathrm{D} p$ and $\mathrm{D} p$-D9 annulus amplitudes and Möbius strip amplitudes in the R-R sectors allows one to recover the spectrum of charged "BPS-like" branes, while combining their contributions reconstructs the dimensions of the adjoint representations of the worldvolume gauge group, namely $\operatorname{USp}(2 N)$ or $\mathrm{SO}(2 N)$. Finally, the coefficients in front of the NS-NS and R-R characters correspond to tension and charge respectively.

The analysis in [20] shows that, similarly to the type I superstring, the USp(32) orientifold model contains charged D1-branes and D5-branes, whose world-volume gauge groups are symplectic and orthogonal respectively. Moreover, the remaining values of $p$ pertain to uncharged branes, whose stability can be addressed studying tachyonic excitations. In particular, a single D3-brane and a single D4-brane are free of tachyons in this model, while a single D0-brane, whose tachyons belong to the adjoint representation of $\operatorname{USp}(2) \simeq \operatorname{SU}(2)$, is unstable. Similarly, a single D2-brane, whose tachyons belong to the anti-symmetric (singlet) representation of $\mathrm{USp}(2)$, is unstable. The other D $p$-branes are unstable on account of bi-fundamental tachyons, arising from the interaction with the background $\overline{\mathrm{D} 9}$-branes.

The spectrum of D-branes in the type $0^{\prime} \mathrm{B}$ model was studied in [20, 47]. The model contains charged $\mathrm{D} p$-branes with $p$ odd, and while their world-volume excitations are devoid of tachyons, D $p$-D $q$ exchanges include tachyons for $|p-q|<4$, analogously to the type IIB setting. Furthermore, once again mirroring the type IIB setting, the world-volume gauge groups are unitary. However, the D9-D7 exchange spectrum contains a tachyon. Even values of $p$ pertain to uncharged branes.

At leading order the $\mathrm{D} p$ - $\mathrm{D} p$ interaction between charged branes vanishes [20], but one is to take into account the presence of the $\overline{\mathrm{D} 9}$-branes and O9-plane, which bring along non-trivial contributions. The main aim of this paper is to investigate this interaction in a number of complementary regimes, and the resulting dynamics appears to realize the WGC in a non-trivial fashion [13] when supersymmetry is broken.

\subsection{Low-energy effective description}

Let us now introduce the low-energy effective description pertaining to the models introduced in section 2. As we have anticipated, both the orientifold models and the heterotic model

\footnotetext{
${ }^{7}$ Some projections allow vanishing or suppressed leading contributions to the vacuum energy [41-46].
} 
can be described, at low energies, by an Einstein-frame action of the form ${ }^{8}$

$$
S=\frac{1}{2 \kappa_{D}^{2}} \int d^{D} x \sqrt{-g}\left(R-\frac{4}{D-2}(\partial \phi)^{2}-V(\phi)-\frac{f(\phi)}{2(p+2) !} H_{p+2}^{2}\right),
$$

where the bosonic fields include a dilaton $\phi$ and a $(p+2)$-form field strength $H_{p+2}=d B_{p+1}$. In the relevant string models $D=10$, while

$$
V(\phi)=T e^{\gamma \phi}, \quad f(\phi)=e^{\alpha \phi},
$$

and their perturbative spectra also include Yang-Mills fields, whose contribution to the action takes the form

$$
S_{\text {gauge }}=-\frac{1}{2 \kappa_{D}^{2}} \int d^{D} x \sqrt{-g}\left(\frac{w(\phi)}{4} \operatorname{Tr} \mathcal{F}_{M N} \mathcal{F}^{M N}\right)
$$

with $w(\phi)$ an exponential. Although AdS compactifications supported by gauge fields of this type were studied in [48], their perturbative corners seem not to exhibit any novel features, and thus we shall neglect this contribution to the EFT action in this paper.

The (bosonic) low-energy dynamics of the orientifold models is described by the Einstein-frame parameters

$$
D=10, \quad p=1, \quad \gamma=\frac{3}{2}, \quad \alpha=1,
$$

while the residual tension

$$
T=2 \kappa_{10}^{2} \times 64 T_{\mathrm{D} 9}=\frac{16}{\pi^{2} \alpha^{\prime}}
$$

in the BSB model reflects the presence of $16 \overline{\mathrm{D} 9}$-branes and the O9-plane [5]. In the type $0^{\prime} \mathrm{B}$ model $T$ is half of this value, since the corresponding O9-plane is tensionless.

The heterotic model is described by the Einstein-frame parameters

$$
D=10, \quad p=1, \quad \gamma=\frac{5}{2}, \quad \alpha=-1,
$$

and the one-loop cosmological constant $T$, which was estimated in [1], is of order one in string units. Dualizing the Kalb-Ramond form one can equivalently work with the parameters

$$
D=10, \quad p=5, \quad \gamma=\frac{5}{2}, \quad \alpha=1,
$$

which highlight the electric coupling of NS5-branes to the dual $B_{6}$ potential.

Let us collect a few remarks on the reliability of the effective action of eq. (2.4). The dilaton potential contains one less power of $\alpha^{\prime}$ with respect to the other terms, and thus its runaway effects are to be balanced by an additional control parameter. The AdS landscapes studied in $[13,48,49]$ achieve this by means of large fluxes, whereby curvature corrections and string loop corrections are expected to be under control. However, in the orientifold models these are R-R fluxes, and thus a world-sheet formulation appears subtle. ${ }^{9}$ In the heterotic model the fluxes are NS-NS, but the dilaton tadpole arises at one-loop level. On the other hand, the flux-less Dudas-Mourad solutions [51] contains regions where string-loop and curvature corrections are expected to be important.

\footnotetext{
${ }^{8}$ Throughout this paper we use the "mostly plus" metric signature.

${ }^{9}$ It is worth noting that world-sheet CFTs on $\mathrm{AdS}_{3}$ backgrounds have been related to $\alpha^{\prime}$-exact WZW models [50], which in principle could be relevant in this case.
} 


\section{Back-reaction of non-supersymmetric branes}

Let us now turn to the gravitational back-reaction of the branes described in section 2.1. The motivation to investigate it is two-fold: on one hand, we would like to understand interactions between two stacks of branes beyond the regime in which both stacks are light. In particular, whenever one of the stacks is parametrically heavier one can expect to describe the interaction replacing the heavy stack with its gravitational back-reaction. On the other hand, large fluxes appear to constitute the most reliable tool to retain control of the low-energy EFT in the presence of gravitational tadpoles [48], and the (near-horizon limit of the) geometries generated by charged branes can realize settings of this type in a natural fashion [13].

\subsection{Back-reaction of extremal branes}

In this section we review the construction of the Toda-like reduced dynamical system for extremal branes, outlining the resulting gravitational back-reaction [13]. The standard procedure for constructing brane solutions $[52,53]$ is hindered by the gravitational tadpole, and the resulting geometries cannot be expressed in closed form [15, 16, 47] (see also [54] for similar considerations on tachyonic type 0 strings) except for special cases [51]. Extremal $p$-branes entail a residual $\mathrm{SO}(1, p) \times \mathrm{SO}(q)$ space-time symmetry, so that in a suitable gauge the most general solution to the field equations stemming from eq. (2.4) takes the form

$$
\begin{aligned}
d s^{2} & =e^{\frac{2}{p+1} v-\frac{2 q}{p} b} d x_{1, p}^{2}+e^{2 v-\frac{2 q}{p} b} d r^{2}+e^{2 b} R_{0}^{2} d \Omega_{q}^{2}, \\
\phi & =\phi(r), \\
H_{p+2} & =\frac{n}{f(\phi)\left(R_{0} e^{b}\right)^{q}} \operatorname{Vol}_{p+2}, \quad \operatorname{Vol}_{p+2}=e^{2 v-\frac{q}{p}(p+2) b} d^{p+1} x \wedge d r,
\end{aligned}
$$

where $r$ is the transverse radial coordinate, $R_{0}$ is an arbitrary reference radius. The resulting equations stem from a constrained Toda-like system $[47,54]$, described by the action

$$
S_{\text {red }}=\int d r\left[\frac{4}{D-2}\left(\phi^{\prime}\right)^{2}-\frac{p}{p+1}\left(v^{\prime}\right)^{2}+\frac{q(D-2)}{p}\left(b^{\prime}\right)^{2}-U\right],
$$

with

$$
U=-T e^{\gamma \phi+2 v-\frac{2 q}{p} b}-\frac{n^{2}}{2 R_{0}^{2 q}} e^{-\alpha \phi+2 v-\frac{2 q(p+1)}{p} b}+\frac{q(q-1)}{R_{0}^{2}} e^{2 v-\frac{2(D-2)}{p} b},
$$

and the Hamiltonian constraint reads

$$
\frac{4}{D-2}\left(\phi^{\prime}\right)^{2}-\frac{p}{p+1}\left(v^{\prime}\right)^{2}+\frac{q(D-2)}{p}\left(b^{\prime}\right)^{2}+U=0 .
$$

Here, the (electric) flux $n$ ought to be proportional to the number $N$ of branes, and is defined by

$$
n=\frac{1}{\Omega_{q}} \int_{\mathbb{S} q} f \star H_{p+2}
$$

with $\Omega_{q}$ the volume of the unit $q$-sphere. Let us remark that eq. (3.5) reflects the brane-like nature of the source, which is supported on the world-volume spanned by the $x$ coordinates. 
Indeed, locally $d\left(f \star H_{p+2}\right)=0$, but its integral in the transverse space yields a finite charge. Similar considerations hold for the energy-momentum tensor, and these sources can be reproduced adding a brane action to eq. (2.4) of the form that we shall employ in section 4.1.

In [13] the $\mathrm{AdS} \times \mathbb{S}$ solutions of [48] were recovered according to

$$
\begin{aligned}
\phi & =\phi_{0}, \\
e^{v} & =\frac{L}{p+1}\left(\frac{R}{R_{0}}\right)^{\frac{q}{p}} \frac{1}{-r}, \\
e^{b} & =\frac{R}{R_{0}},
\end{aligned}
$$

where $r<0$. Anticipating that this solution arises as a near-horizon limit, this choice places the core at $r \rightarrow-\infty$. Rescaling $x$ by a constant, and substituting

$$
r \mapsto-\frac{z^{p+1}}{p+1}
$$

in the metric of eq. (3.1) reveals that the solution in eq. (3.6) is indeed $\operatorname{AdS} \times \mathbb{S}$ in a Poincaré patch. Following the analogy with the supersymmetric cases of the four-dimensional ReissnerNordström black hole and type IIB D3-brane stacks, where infinite AdS throats behave as attractors, in [13] it was shown that radial perturbations always contain modes that decay going toward the horizon, while the blow-up modes are expected to be associated to extremality breaking. While control of the full solution appears necessary to connect the asymptotic parameters to these modes, one can verify that they reassuringly match in number studying the sub-leading behavior in the far-away region. Indeed, while we shall not need this result in the following, it turns out that the back-reacted geometry ends, at a finite radial geodesic distance, in a pinch-off singularity where strong-coupling effects are expected to play a crucial rôle. The resulting suggestive, albeit incomplete, picture is presented in figure 1, and highlights the obstructions in defining tension and flux as asymptotic quantities. The non-extremal version of this construction contains an additional blackening factor [55], but the pinch-off singularity appears to be universal [56] and has been recently connected to some swampland conjectures [39, 40].

\subsubsection{Nucleation in AdS and brane tension}

The AdS $\times \mathbb{S}$ near-horizon solution of eq. (3.6) can be recast in the coordinate-free fashion

$$
\begin{aligned}
d s^{2} & =L^{2} d s_{\mathrm{AdS}_{p+2}}^{2}+R^{2} d \Omega_{q}^{2}, \\
H_{p+2} & =c \operatorname{Vol}_{\mathrm{AdS}_{p+2}}, \\
\phi & =\phi_{0},
\end{aligned}
$$

where $d s_{\mathrm{AdS}_{p+2}}^{2}$ is the unit-radius space-time metric and $\operatorname{Vol}_{\mathrm{AdS}_{p+2}}$ denotes the canonical volume form on $\mathrm{AdS}_{p+2}$ with radius $L$. The geometry exists if and only if the parameters $\gamma, \alpha$ in eq. (2.5) satisfy

$$
\alpha>0, \quad q>1, \quad(q-1) \gamma>\alpha
$$




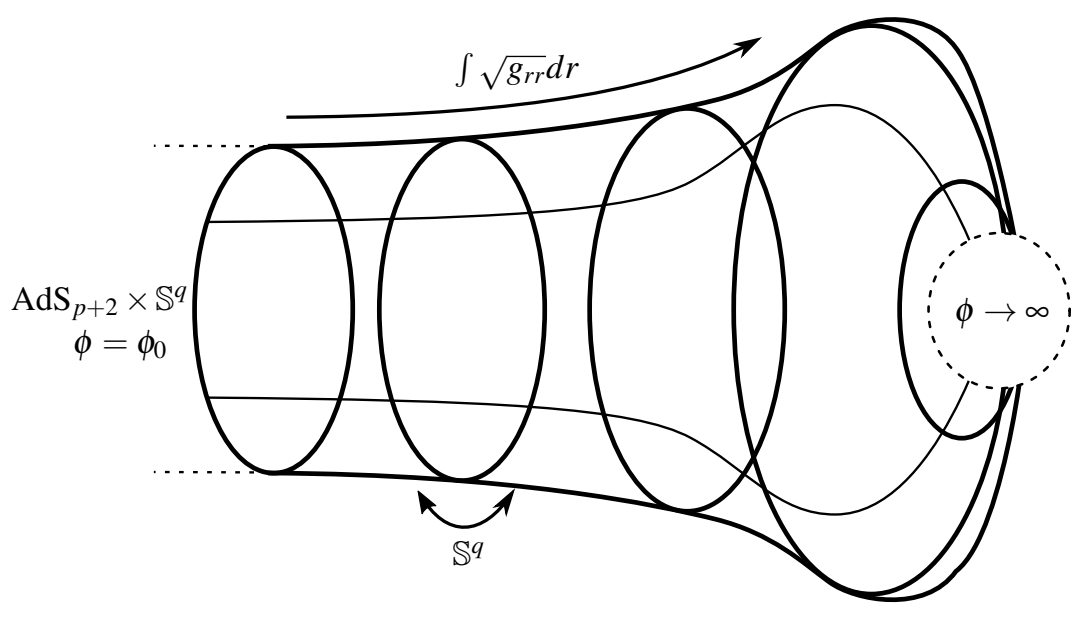

Figure 1. a schematic depiction of the back-reacted geometry sourced by the branes, where the world-volume directions are excluded. The geometry interpolates between the AdS $\times \mathbb{S}$ throat and the pinch-off singularity.

and using eq. (2.5) the string coupling $g_{s}=e^{\phi_{0}}$ and the curvature radii $L, R$ are given by

$$
\begin{aligned}
c & =\frac{n}{g_{s}^{\alpha} R^{q}}, \\
g_{s}^{(q-1) \gamma-\alpha} & =\left(\frac{(q-1)(D-2)}{\left(1+\frac{\gamma}{\alpha}(p+1)\right) T}\right)^{q} \frac{2 \gamma T}{\alpha n^{2}}, \\
R^{2^{\frac{(q-1) \gamma-\alpha}{\gamma}}} & =\left(\frac{\alpha+(p+1) \gamma}{(q-1)(D-2)}\right)^{\frac{\alpha+\gamma}{\gamma}}\left(\frac{T}{\alpha}\right)^{\frac{\alpha}{\gamma}} \frac{n^{2}}{2 \gamma}, \\
L^{2} & =R^{2}\left(\frac{p+1}{q-1} \cdot \frac{(p+1) \gamma+\alpha}{(q-1) \gamma-\alpha}\right) .
\end{aligned}
$$

The solution of eq. (3.10), originally found in [48] for the string models presented in section 2, was generalized and studied in detail in [13, 49]. Among its intriguing features, it appears complementary to the supersymmetric $\operatorname{AdS}_{5} \times \mathbb{S}^{5}$ solution of type IIB supergravity, but it has no moduli. Moreover, the large- $n$ limit corresponds both to small string couplings and small curvatures, and thus one can expect that the EFT description encoded in eqs. (2.7) and (2.10) be reliable in this regime. However, let us emphasize that a dimensionally reduced EFT description would not be consistent, since there is no scale separation. This is consistent with general considerations on scale separation $[57,58],{ }^{10}$ while the behavior of Kaluza-Klein excitations corroborates the Swampland distance conjecture (SDC) [60, 61] and AdS distance conjecture (ADC) [57]. The connection between the non-supersymmetric backgrounds that we have discussed and Swampland conjectures, in particular those regarding dS vacua [61-63], has been articulated in detail in [14].

In light of the preceding discussion, the $\operatorname{AdS} \times \mathbb{S}$ solutions of eq. (3.10) appear to capture the near-horizon back-reaction of extremal branes in the presence of gravitational tadpoles. Specifically, they pertain to D1-branes in the orientifold models and NS5-branes in the

\footnotetext{
${ }^{10}$ See also [59] for a detailed study of bounds on scale separation in flux compactifications.
} 
heterotic model, while an analogous solution describing extremal D5-branes is still lacking and presents some subtleties. ${ }^{11}$ Although the internal sphere ought to reflect the simplest setting of parallel branes in the vacuum, it brings along perturbative instabilities [49]. Since eq. (3.10) readily generalizes to any compact Einstein manifold, in principle it could be possible to eliminate the unstable modes choosing a different internal manifold, possibly pertaining to branes on conical singularities, and for the heterotic model an antipodal $\mathbb{Z}_{2}$ orbifold of the internal $\mathbb{S}^{3}$ indeed achieves this. In this paper we shall thus neglect these instabilities.

However, these solutions are also fraught with non-perturbative instabilities, and undergo flux tunneling [13 $]^{12}$ in accord with the non-supersymmetric AdS Swampland conjecture [60]. In particular, in [13] it was shown that the semi-classical decay rate per unit volume of the AdS solutions is given by the exponentiated (extremized) instanton action of a brane. Furthermore, consistency with the semi-classical limit requires that the string-frame tension $\tau_{p}=T_{p} e^{-\sigma \phi}$ of the brane scale with the dilaton according to

$$
\sigma=\frac{2(p+1)}{D-2}+\frac{\alpha}{2}=1+\frac{\alpha_{S}}{2}
$$

where $\alpha_{S}$ is the string-frame counterpart of the parameter $\alpha$ in eq. (2.5). This result remarkably reproduces the correct couplings of fundamental branes, namely D-branes in the orientifold models, NS5-branes in the heterotic model and the "exotic" branes of [73-77], thereby further suggesting that the branes sourcing the AdS throat and nucleating in it are indeed D1-branes in the orientifold models and NS5-branes in the heterotic model. As we shall see shortly, the fact that brane nucleation occurs also entails a novel realization of the WGC in the presence of gravitational tadpoles.

\subsection{Static Dudas-Mourad solutions as 8-branes}

As described in [13], at least in the orientifold models the Dudas-Mourad solution [51] resonates with the back-reaction of D8-branes. This is because the Toda-like dynamical system that we have discussed, along with its non-extremal version [55], describe a general family of $p$-brane solutions, and the $p=8$ case yields an integrable system which matches the Dudas-Mourad solution. In detail, they comprise static solutions with ninedimensional Poincaré symmetry, ${ }^{13}$ where one dimension is compactified on an interval, and ten-dimensional cosmological solutions. In the ensuing discussion we shall focus on the former, in light of our preceding considerations.

The gravitational tadpole signals the absence of a ten-dimensional Minkowski solution, and therefore the maximal possible symmetry available to static solutions is nine-dimensional Poincaré symmetry, compatibly with the presence of 8-brane sources that break its tendimensional counterpart. Correspondingly, the most general solution of this type is a warped product of nine-dimensional Minkowski space-time, parametrized by coordinates $x^{\mu}$, and a

\footnotetext{
${ }^{11}$ This state of affairs mirrors, to a certain extent, the peculiar near-horizon geometry sourced by BPS D5-branes, since it breaks the pattern of conformal $\mathrm{AdS}_{p+2} \times \mathbb{S}^{8-p}$ throats $[52,53,64,65]$.

${ }^{12}$ For more details, see [66-72].

${ }^{13} \mathrm{~A}$ T-dual version of this configuration in the USp(32) model was investigated in [78].
} 
one-dimensional internal space, parametrized by a coordinate $y$. In the orientifold models of section 2, the Einstein-frame solution is given by

$$
\begin{aligned}
d s_{\text {orientifold }}^{2} & =\left|\alpha_{\mathrm{O}} y^{2}\right|^{\frac{1}{18}} e^{-\frac{\alpha_{\mathrm{O}} y^{2}}{8}} d x_{1,8}^{2}+e^{-\frac{3}{2} \Phi_{0}}\left|\alpha_{\mathrm{O}} y^{2}\right|^{-\frac{1}{2}} e^{-\frac{9 \alpha_{\mathrm{O}} y^{2}}{8}} d y^{2}, \\
\phi & =\frac{3}{4} \alpha_{\mathrm{O}} y^{2}+\frac{1}{3} \log \left|\alpha_{\mathrm{O}} y^{2}\right|+\Phi_{0}
\end{aligned}
$$

where, here and in the following,

$$
d x_{1, p}^{2} \equiv \eta_{\mu \nu} d x^{\mu} d x^{\nu}
$$

denotes the $(p+1)$-dimensional Minkowski metric. The absolute values in eq. (3.12), as well as the singularities in the dilaton profile, highlight that the physical range of the internal coordinate is $y \in(0, \infty)$. The heterotic model affords an analogous solution, and similar considerations on the range of $y$ apply.

In accord with the conventions of [51], in eqs. (3.12) the scale $\alpha_{\mathrm{O}} \equiv \frac{T}{2}$, while $\Phi_{0}$ is a free parameter which specifies the value of the local string coupling at some $y=y_{0}$. In both solutions, the internal spaces parametrized by $y$ are actually intervals of finite length

$$
R_{c} \equiv \int_{0}^{\infty} \sqrt{g_{y y}} d y<\infty
$$

and, for $g_{s} \equiv e^{\Phi_{0}} \ll 1$, the interior of the parametrically wide interval is weakly coupled. Moreover, since $\alpha_{\mathrm{O}} \propto T$, as one approaches the supersymmetric case the internal length diverges. ${ }^{14}$

\subsection{D3-branes in the type $0^{\prime} \mathrm{B}$ model}

As we have discussed, the AdS $\times \mathbb{S}$ solutions that we have reviewed in the preceding section appear in some sense complementary to the supersymmetric type IIB setting. Indeed, for $p \neq 3,5$ the near-horizon geometry of BPS black branes in ten dimensions is conformal to $\mathrm{AdS}_{p+2}$ with a singular warp factor [52, 53, 64, 65], while for $p=3$ (and for M-branes in eleven-dimensional supergravity) it reproduces the familiar $\mathrm{AdS}_{p+2}$. On the other hand, in the models that we have presented in section 2 there are no such solutions, since $\alpha=0$ for D3-branes in the type $0^{\prime} \mathrm{B}$ model. The relevant near-horizon geometry that was studied in $[15,16,47]$ actually involves an O3-plane, but its contribution is sub-leading for large fluxes. In [16] the authors found non-homogeneous deviations from $\mathrm{AdS}_{5} \times \mathbb{R} \mathbb{P}^{5}$ which are suppressed, but not uniformly so, in the large-flux limit. ${ }^{15}$ In detail, in coordinates in which the (string-frame) metric takes the form ${ }^{16}[16]$

$$
d s^{2}=R^{2}(u) \frac{d u^{2}}{u^{2}}+\frac{\alpha^{\prime 2} u^{2}}{R^{2}(u)} d x_{1,3}^{2}+\widetilde{R}^{2}(u) d \Omega_{5}^{2},
$$

\footnotetext{
${ }^{14}$ Let us remark that, strictly speaking, $T$ cannot be sent to zero, but it is still instructive to consider the formal limit $T \rightarrow 0$.

${ }^{15}$ Similar results in tachyonic type 0 strings were obtained in [54].

${ }^{16}$ The local expression in eq. (3.15) does not account for the global distinction between $\mathbb{S}^{5}$ and $\mathbb{R} \mathbb{P}^{5}$.
} 
the would-be $\mathrm{AdS}_{5}$ and $\mathbb{R} \mathbb{P}^{5}$ curvature radii $R(u), \widetilde{R}(u)$ and the dilaton $\phi(u)$ acquire a dependence on the energy scale $u$ that, in the large-flux limit, behaves as

$$
\begin{aligned}
\frac{R^{2}(u)}{R_{\infty}^{2}} & \sim 1-\frac{3}{16} g_{s} \alpha^{\prime} T \log \left(\frac{u}{u_{0}}\right), \\
\frac{\widetilde{R}^{2}(u)}{R_{\infty}^{2}} & \sim 1-\frac{3}{16 \sqrt[4]{8}} g_{s}^{2} N \alpha^{\prime} T \log \left(\frac{u}{u_{0}}\right), \\
\frac{1}{N} e^{-\phi} & \sim \frac{1}{g_{s} N}+\frac{3}{8 \sqrt[4]{8}} g_{s} \alpha^{\prime} T \log \left(\frac{u}{u_{0}}\right),
\end{aligned}
$$

where $u_{0}$ is a reference scale, $R_{\infty}^{2}=\sqrt{4 \pi g_{s} N}$ is the supersymmetric value of the radii and $N \gg 1$ ought to be interpreted as the number of D3-branes sourcing the geometry. In the large- $N$ limit the 't Hooft coupling $\lambda=4 \pi g_{s} N$ in the absence of the tadpole $T$ ought to be fixed, but the validity of the EFT description also requires $\lambda \gg 1$ [79], while on account of the second of eq. (3.16) $g_{s}^{2} N \ll 1$.

Although the resulting geometry is not completely under control because of the nonuniform character of these corrections, it is in principle amenable to numerical investigation via the Toda-like formalism that we have developed in section 3.1 [47]. In the following section we shall discuss the interactions of D3-branes in the type $0^{\prime} \mathrm{B}$ model in the regime in which a heavy stack generates the solution of eq. (3.16), and probe light stacks are subjects to a potential encoding the interaction.

Let us conclude this section briefly summarizing how the branes and geometries that we have discussed are related. To begin with, the longitudinal and transverse dimensions match, as the corresponding isometry groups. Furthermore, the type of fluxes, if any, also match, and the solutions all arise from a single family of Toda-like dynamical systems that reproduce the Dudas-Mourad solutions as a special case. Going beyond the background geometry, the AdS throats exhibit both an attractor-like mechanism, typical of near-horizon limits, and non-perturbative brane nucleation, whose semi-classical consistency requires precisely the tensions pertaining to the fundamental branes at stake. Although the absence of supersymmetry hinders the extrapolation of these results beyond the EFT regime to some extent, the overall picture appears coherent, and we shall explore the resulting lessons for the dynamics of branes in the remainder of this paper.

\section{Brane interactions and the WGC}

In this section we study in detail the interactions between the branes that we have introduced in section 2.1. To this end, we shall discuss a number of complementary regimes in which computations are expected to be under control. Namely, considering two parallel stacks of $N_{p} \mathrm{D} p$-branes and $N_{q} \mathrm{D} q$-branes, the cases that we shall address are the following:

- The probe regime $N_{p} \gg N_{q}$, in which one can replace the heavy stack of $N_{p} \mathrm{D} p$-branes with the corresponding back-reacted geometry probed by the $\mathrm{D} q$-branes, as shown in figure 2. We shall distinguish three cases: extremal branes probing AdS throats, uncharged D8-branes probing AdS throats, D $p$-branes probing the Dudas-Mourad geometry sourced by D8-branes. We shall also address NS5-branes probing the heterotic 
Dudas-Mourad geometry for completeness, although in that case an interpretation in terms of 8-branes appears obscure in the absence of established dualities.

- The string-amplitude regime $N_{p}, N_{q}=\mathcal{O}(1)$, which ought to be described by perturbative string amplitudes. In particular, since interactions between extremal branes yield vanishing annulus amplitudes, we shall focus on the case in which at least one stack is uncharged. ${ }^{17}$

- The holographic regime, in which we shall consider the asymptotically free worldvolume gauge theory that describes D1-branes. In this paper we shall focus on the weakly coupled UV regime, although the strongly coupled IR regime ought to be directly related to near-horizon AdS throats. We shall discuss this interesting case in future work.

Despite the absence of supersymmetry, whenever any two regimes overlap we shall find qualitative agreement. In particular, extremal branes of equal dimension strictly repel, realizing the WGC in the absence of supersymmetry, while the NS-NS interactions in the presence of at least one uncharged stack are repulsive or attractive depending on the values of $p$ and $q$. To wit, in section 4.1 we compute static potentials for extremal $\mathrm{D} p$-branes in the orientifold models (resp. NS5-branes in the heterotic model) probing the geometries sourced by a heavy stack of parallel $\mathrm{D} p$ branes (resp. NS5-branes in the heterotic model), according to the evidence presented in the preceding section, finding a repulsive behavior and a $\mathcal{O}(1)$ verification of the WGC bound. Then, we compute static potentials for $\mathrm{D} p$-branes probing the Dudas-Mourad geometry, which according to our arguments ought to sourced by D8-branes, and for 8-branes probing the AdS throats presented in section 3. We compare our results with the string-amplitude computations of section 4.2, finding qualitative agreement for repulsive and attractive behaviors. Finally, in section 4.3 we address D1-branes holographically computing the one-loop effective potential of the world-volume gauge theory.

\subsection{Probe potentials and Weak Gravity}

Let us begin analyzing the probe regime, replacing the heavy brane stack with its backreacted geometry. In particular we shall focus on the near-horizon $\mathrm{AdS} \times \mathbb{S}$ throats that we have described in section 3.1.1, which ought to pertain to D1-branes and NS5-branes, and on the Dudas-Mourad geometry, which appears to arise from 8-branes. In order to encompass all the relevant cases, we shall consider a string-frame world-volume action of the form

$$
S_{p}=-T_{p} \int d^{p+1} \zeta \sqrt{-j^{*} g_{S}} e^{-\sigma \phi}+\mu_{p} \int B_{p+1},
$$

where $j$ is the embedding of the world-volume coordinates $\zeta$ in space-time. Its Einstein-frame expression reads

$$
S_{p}=-T_{p} \int d^{p+1} \zeta \sqrt{-j^{*} g} e^{\left(\frac{2(p+1)}{D-2}-\sigma\right) \phi}+\mu_{p} \int B_{p+1},
$$

\footnotetext{
${ }^{17}$ The first non-trivial contribution to the potential between two extremal stacks would involve three-legged pants diagrams. In the bosonic case, the systematics of such computations were developed in [80].
} 


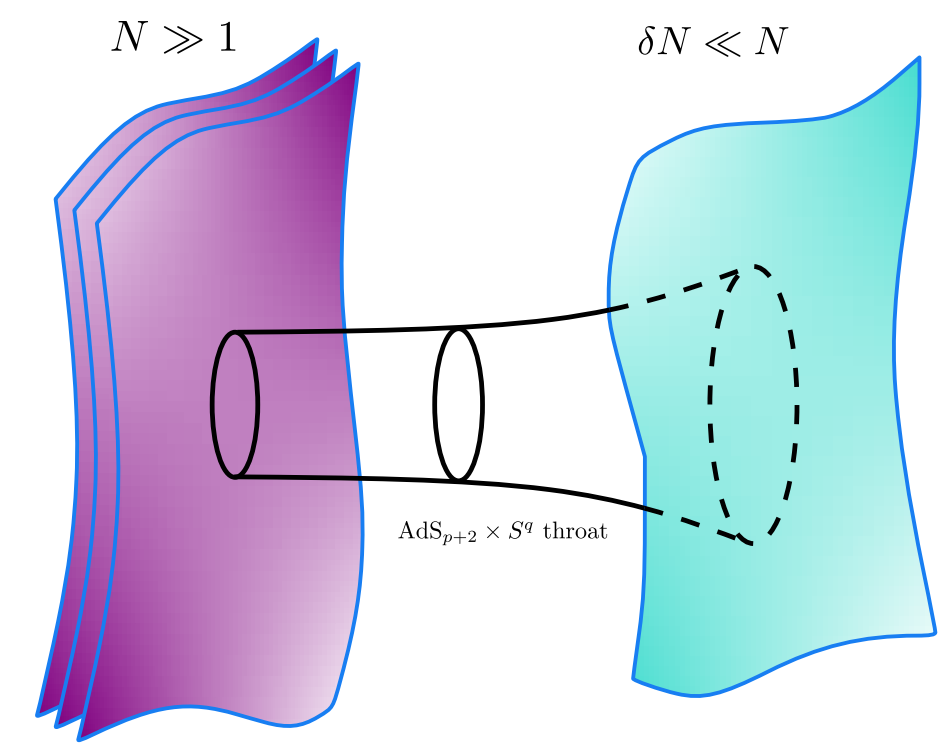

Figure 2. a depiction of the interaction between a heavy stack of $N \gg 1$ branes and $\delta N \ll N$ probe branes. The heavy stack sources the $\operatorname{AdS} \times \mathbb{S}$ throat probed by the light stack.

and $\sigma=1,2$ for D-branes and NS5-branes respectively. For the sake of generality we shall not assume that $T_{p}=\mu_{p}$ in the non-supersymmetric models at stake, although the results of [20] point toward extremality. However, as we shall now see in detail, in the string models that we have presented in section 2 even in this case charged brane stacks repel, due to a non-trivial renormalization of the charge-to-tension ratio mediated by supersymmetry breaking.

\subsubsection{Repulsive forces between extremal branes}

Let us begin our analysis of probe-brane interactions considering the dynamics of an extremal $p$-brane moving in the $\operatorname{AdS}_{p+2} \times \mathbb{S}^{q}$ geometry of eq. (3.8). The action in eq. (4.2) encompasses both D1-branes in the orientifold models, where $B_{2}$ is a R-R potential, and NS5-branes in the heterotic model, where $B_{6}$ is the (magnetic dual of the) Kalb-Ramond potential. As we have stressed in the preceding section, the putative electromagnetic dual of these background would describe D5-branes and F1-strings, but their construction has proven elusive so far.

Let us remark that the dynamics at stake emerge spontaneously on account of the considerations in section 3.1.1, since bubble nucleation entails separation of pairs of branes and anti-branes. The results in [13] show that like-charge branes are repelled while antibranes are attracted, leading to brane-flux annihilation. In order to appreciate this, it is convenient to work in Poincaré coordinates, where the Einstein-frame metric of the $\operatorname{AdS} \times \mathbb{S}$ throat reads

$$
d s^{2}=\frac{L^{2}}{z^{2}}\left(d z^{2}+d x_{1, p}^{2}\right)+R^{2} d \Omega_{q}^{2}
$$

choosing the world-volume embedding

$$
j: \quad x^{\mu}=\zeta^{\mu}, \quad z=Z(\zeta), \quad \theta^{i}=\theta_{0}^{i}
$$


with $\theta_{0}^{i}$ fixed coordinates on $\mathbb{S}^{q}$. The action of eq. (4.2) then evaluates to

$$
S_{p}=-\tau_{p} \int d^{p+1} \zeta\left(\frac{L}{Z}\right)^{p+1}\left[\sqrt{1+\eta^{\mu \nu} \partial_{\mu} Z \partial_{\nu} Z}-\frac{c L}{p+1} \frac{\mu_{p}}{\tau_{p}}\right],
$$

where the dressed tension

$$
\tau_{p} \equiv T_{p} g_{s}^{-\frac{\alpha}{2}}
$$

on account of the considerations of section 3.1.1. Therefore, rigid branes are subject to the potential

$$
\begin{aligned}
V_{\text {probe }}(Z) & =\tau_{p}\left(\frac{L}{Z}\right)^{p+1}\left[1-\frac{c L g_{s}^{\frac{\alpha}{2}}}{p+1} \frac{\mu_{p}}{T_{p}}\right] \\
& =\tau_{p}\left(\frac{L}{Z}\right)^{p+1}\left[1-v_{0} \frac{\mu_{p}}{T_{p}}\right]
\end{aligned}
$$

where the $\mathcal{O}(1)$ constant $v_{0}>1$ in our string models [13]. While non-rigid branes exhibit richer dynamics, in the present setting we would like to emphasize that, since crucially $v_{0}>1$, extremal probes $\mu_{p}=T_{p}$ are indeed repelled by the stack, being driven toward $Z \rightarrow 0$. In the orientifold models the picture is intuitive: D1-branes are mutually BPS, ${ }^{18}$ but their interaction with the space-time-filling $\overline{\mathrm{D} 9}$-branes and O9-plane renormalizes the charge-totension ratio, as depicted in figure 3. Similarly, in the heterotic model the corresponding interactions are mediated by the quantum-corrected vacuum energy. This peculiar result realizes the WGC in this particular setting in a non-trivial fashion: while brane nucleation in non-supersymmetric AdS has been thoroughly investigated [60, 68, 69], let us stress that in the present case this phenomenon arises from extremal branes interacting in the absence of supersymmetry.

To conclude this section, let us consider probe-regime interactions between D3-branes, whose corresponding near-horizon throat deviates from AdS $\times \mathbb{S}$ as we have discussed in section 3.3. Indeed, since the EFT parameter $\alpha=0$ in this case, an $\operatorname{AdS} \times \mathbb{S}$ solution cannot exist. For more details on the back-reacted geometry, see [16]. Our starting point is now the solution in eqs. (3.15) and (3.16). Let us once again embed the probe world-volume parallel to the $x^{\mu}$, according to

$$
j: \quad x^{\mu}=\zeta^{\mu}, \quad u=\mathrm{U}(\zeta), \quad \theta^{i}=\theta_{0}^{i},
$$

where the coordinate $u$ is to be interpreted as an energy scale.

The five-form R-R field strength $F_{5}$ is self-dual, closed, ${ }^{19}$ and reads

$$
\begin{aligned}
F_{5} & =(1+\star) f_{5} N \operatorname{vol}_{\mathbb{S}^{5}} \\
& =f_{5} N \operatorname{vol}_{\mathbb{S}^{5}}+\frac{f_{5} N}{\widetilde{R}(u)^{5}}\left(\frac{\alpha^{\prime} u}{R(u)}\right)^{3} d\left(\alpha^{\prime} u\right) \wedge d^{4} x
\end{aligned}
$$

\footnotetext{
${ }^{18}$ More precisely, despite the absence of supersymmetry, in the type $0^{\prime} \mathrm{B}$ model charged branes exhibit a "BPS-like" no-force behavior at tree-level [20].

${ }^{19}$ Since the orientifold projection removes the Kalb-Ramond form, no additional terms appear in the Bianchi identity.
} 


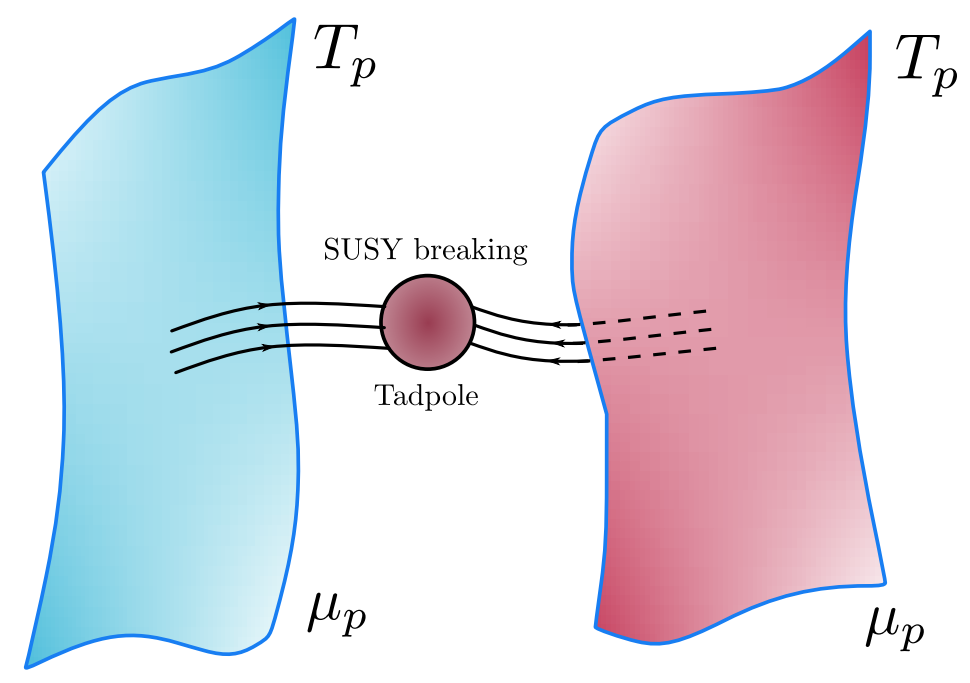

Figure 3. a depiction of the interaction between extremal branes mediated by supersymmetry breaking, reflecting the renormalization of the effective charge-to-tension ratio $v_{0}$ of eq. (4.7).

with $\operatorname{vol}_{\mathbb{S}^{5}}$ the volume form of the unit 5 -sphere. The flux quantization condition

$$
\frac{1}{2 \kappa_{10}^{2}} \int_{\mathbb{S}^{5}} F_{5}=\mu_{3} N
$$

then fixes

$$
f_{5}=\frac{2 \kappa_{10}^{2} \mu_{3}}{\Omega_{5}},
$$

and the relevant contribution to the potential $C_{4}$, to be pulled back on the probe worldvolume, takes the form

$$
C_{4}=c_{4}(u) d^{4} x+\ldots
$$

where $d C_{4}=F_{5}$ implies

$$
\frac{c_{4}^{\prime}(u)}{\alpha^{\prime}}=\frac{f_{5} N}{\widetilde{R}(u)^{5}}\left(\frac{\alpha^{\prime} u}{R(u)}\right)^{3} .
$$

Collecting all the ingredients, and using the string-frame world-volume action of eq. (4.1), the probe potential evaluates to

$$
V_{\text {probe }}^{\mathrm{D} 3}(U)=T_{3}\left(\frac{\alpha^{\prime} U}{R(U)}\right)^{4} e^{-\phi(U)}-\mu_{3} c_{4}(U),
$$

and its dominant contribution in the EFT limit $g_{s}, g_{s}^{2} N \ll 1, g_{s} N \gg 1$ is

$$
\begin{aligned}
\frac{V_{\text {probe }}^{\text {D3 }}(U)}{U^{4}} \sim & \frac{16 \pi \alpha^{\prime 2} T_{3}-f_{5} \mu_{3}}{64 \pi^{2} g_{s}^{2} N}+\frac{15 f_{5} \mu_{3} \alpha^{\prime} T}{8192 \sqrt[4]{8} \pi^{2}} \\
& +\frac{3\left(64 \pi \alpha^{\prime 2} T_{3}-5 f_{5} \mu_{3}\right) \alpha^{\prime} T}{2048 \sqrt[4]{8} \pi^{2}} \log \left(\frac{U}{u_{0}}\right) .
\end{aligned}
$$

As expected, substituting the supersymmetric values

$$
2 \kappa_{10}^{2}=(2 \pi)^{7} \alpha^{\prime 4}, \quad T_{3}=\mu_{3}=\frac{N_{3}}{(2 \pi)^{3} \alpha^{\prime 2}}
$$




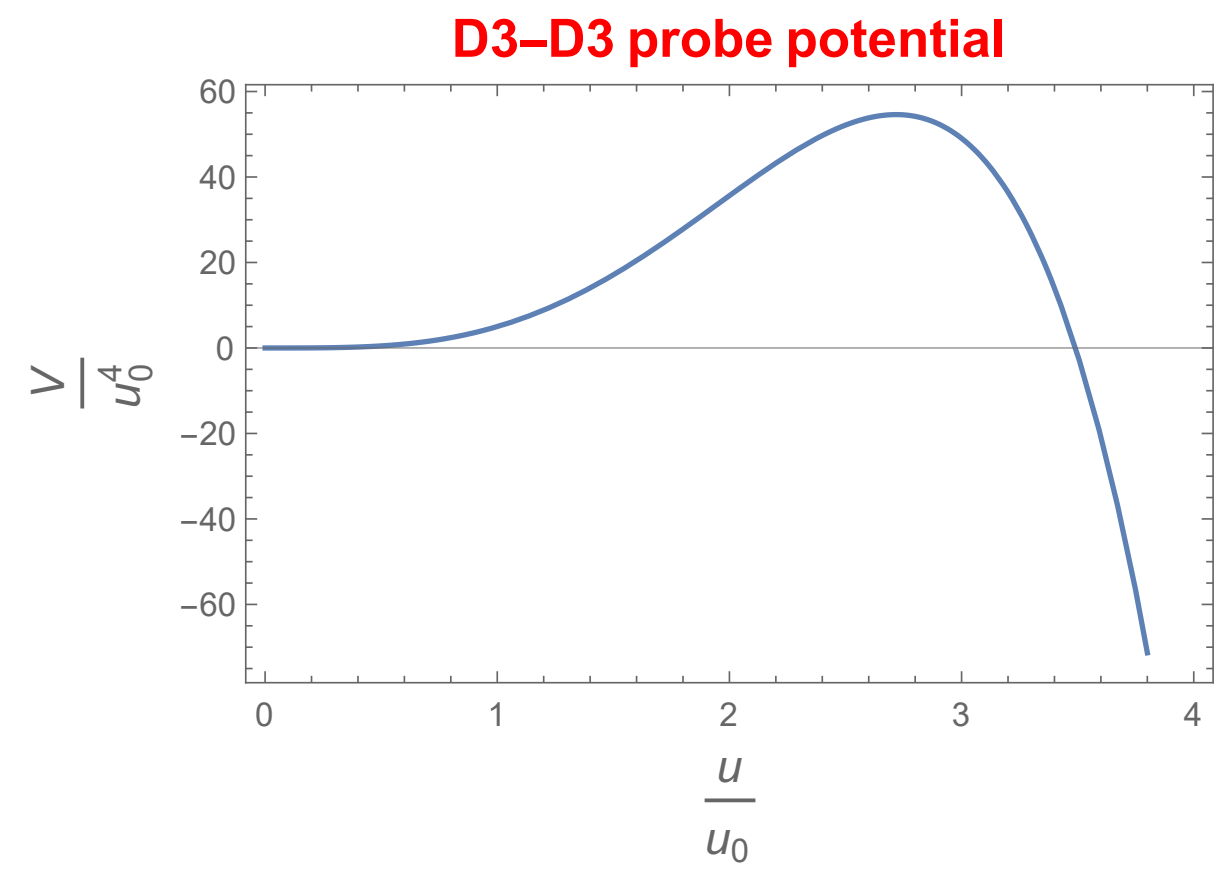

Figure 4. the normalized probe potential in eq. (4.15) in units of the reference scale $u_{0}$.

for $N_{3} \ll N$ probes, and using eq. (4.11), the leading term vanishes, on account of the BPS property, while the remaining sub-leading terms reflect supersymmetry breaking and their $U$-dependence simplifies to

$$
V_{\text {sub-leading }}^{\text {D3 }}(U) \propto U^{4}\left[5-4 \log \left(\frac{U}{u_{0}}\right)\right] .
$$

Once again this potential is repulsive, realizing the WGC also in this "marginal" setting, since $U \rightarrow \infty$ corresponds to exiting the throat. Interestingly, as depicted in figure 4 , the potential in eq. (4.17) features a maximum at $U=e u_{0}$ before crossing zero at $U=e^{\frac{5}{4}} u_{0}$, and the height of the potential barrier scales proportionally to $u_{0}^{4}$. Therefore, even if the probe stack were initially located in the classically attractive region, it would eventually tunnel to the repulsive region. Indeed, for suitably small velocities $\alpha^{\prime} \partial_{t} U \ll 1$, the kinetic term in the DBI action is asymptotic to

$$
\frac{\alpha^{\prime 2} T_{3}}{2 g_{s}}\left[1+\frac{3 \alpha^{\prime} T}{8 \sqrt[4]{8}} g_{s}^{2} N \log \left(\frac{U}{u_{0}}\right)\right]\left(\partial_{t} U\right)^{2},
$$

and since the potential is purely sub-leading the canonical field redefinition would not modify its dominant contribution. Furthermore, let us recall that the geometry in eq. (3.16) has a limited regime of validity, which we expect to extend at most up to $\frac{u}{u_{0}}=\mathcal{O}\left(e^{N}\right)$, where sensible dynamics is expected to replace the unbounded approximate potential of eq. (4.15). A potentially instructive toy model, in which the unknown large- $u$ dynamics is substituted by a hard wall at some cut-off scale $u=\Lambda$, would then feature an asymmetric double-well, which can result in bubble nucleation [81, 82]. 
As we have discussed in the preceding section, an attempt to reproduce these results via a string amplitude computation, at least for the orientifold models, would be met by considerable difficulties, since the relevant annulus contribution vanishes. On the other hand, in the non-extremal case one has access both to the gravitational back-reaction of D8-branes and to a string amplitude computation, and thus we shall turn to this issue in section 4.1.2.

As a final comment, let us observe that in the heterotic model one can also compute the potential for probe F1-strings, extended along one of the directions parallel to the NS5-branes. However, the Kalb-Ramond form $B_{2}$ vanishes upon pull-back on the string world-sheet, and thus the resulting force is attractive. The counterpart of this setting in the orientifold models would involve probe D5-branes, but these would wrap contractible cycles on the spheres, leading to an uncontrolled computation.

\subsubsection{Brane probes in the Dudas-Mourad geometry}

Let us now extend the considerations of the preceding section to the case in which at least one of the two brane stacks is uncharged. While this case is not directly relevant for the WGC, it is instructive to compare the resulting dynamics to string amplitude computations in the absence of supersymmetry. Indeed, with respect to the extremal case, the leading contribution to the relevant string amplitude corresponds to annulus, which does not vanish and does not entail Riemann surfaces of higher Euler characteristic and other complications. In particular, we shall focus on D8-branes in the orientifold models, since their back-reacted geometry is described by the static Dudas-Mourad solution ${ }^{20}[51]$ that we have described in section 3.2 .

Furthermore, the other controlled back-reacted geometry in this setting corresponds to D1-branes, and D8-branes are the only other probes whose potential can be reliably computed in this case, since they can wrap the $\mathbb{S}^{7}$ in the near-horizon $\mathrm{AdS}_{3} \times \mathbb{S}^{7}$ throat. On the other hand, as we have discussed, while similar considerations apply to the heterotic model a microscopic interpretation appears more subtle. Nevertheless, probe-brane calculations in this setting yield attractive potentials for 8-branes and fundamental strings, as in the orientifold models, while NS5-branes are repelled. In addition, in some cases the potential scales with a positive power of $g_{s}$. At any rate, the instability appears to be under control, since probes would reach the strong-coupling regions in a parametrically large time for $g_{s} \ll 1$.

To begin with, we shall consider a stack of $N_{p} \mathrm{D} p$-branes probing the Dudas-Mourad geometry. According to the considerations in the preceding sections, this ought to describe a stack of $\mathrm{D} p$-branes parallel to a stack of D8-branes. ${ }^{21}$ In order to simplify the ensuing discussion, we work in the string frame in units where $\alpha_{\mathrm{O}}=1$. Since the boundary of the interval spanned by the coordinate $y$ hosts two singularities, we expect this configuration to

\footnotetext{
${ }^{20}$ The generalization to non-extremal branes of different dimensions involves non-integrable Toda-like systems [55], whose correct boundary conditions are not well-understood hitherto. In addition, a reliable probe regime would exclude the pinch-off asymptotic region, thereby requiring numerical computations.

${ }^{21}$ By analogy with the results in [13], the number $N_{8}$ of D8-branes, should be implicitly determined by the only free parameter $g_{s} \equiv e^{\Phi_{0}}$ in eq. (3.12), with $g_{s} \ll 1$ for $N_{8} \gg 1$.
} 
be under control insofar as the (string-frame) geodesic coordinate

$$
r \equiv \frac{1}{\sqrt{g_{s}}} \int_{0}^{y} \frac{d u}{u^{\frac{1}{3}}} e^{-\frac{3}{8} u^{2}}
$$

is far away from its endpoints $r=0, r=R_{c}$. This overlap regime indeed exists, provided that $g_{s} \equiv e^{\Phi_{0}} \ll 1$.

Defining the (string-frame) warp factors $A(y), B(y)$ of [51] according to

$$
d s_{10}^{2}=e^{2 A(y)} d x_{9}^{2}+e^{2 B(y)} d y^{2},
$$

the probe action for $\mathrm{D} p$-branes reduces to the DBI action in the absence of fluxes, and it evaluates to

$$
\begin{aligned}
S_{p} & =-N_{p} T_{p} \int d^{p+1} x e^{(p+1) A(y)-\Phi(y)} \\
& \equiv-N_{p} T_{p} \int d^{p+1} x V_{p 8},
\end{aligned}
$$

where we have defined the potential per unit tension

$$
V_{p 8}=g_{s}^{\frac{p-3}{4}} y^{\frac{2}{9}(p-2)} e^{\frac{p-5}{8} y^{2}} .
$$

The non-trivial dependence of eq. (4.22) on $p$ is depicted in figures 5 and 6 . If the potential drives probes toward $y \rightarrow \infty$ it is repulsive, since the pinch-off this regime hosts the pinch-off singularity discussed in [13]. As a result, for $p<3$ probes are repelled by the D8-branes, while for $p>4$ they are attracted to the D8-branes. The cases $p=3,4$ exhibit unstable equilibria, but at large separations the potentials are repulsive. This is the regime that we shall compare with a string amplitude computation.

As we have anticipated, branes probing the back-reacted geometry sourced by nonextremal $p$ branes, with $p<8$, entail considerable difficulties. Indeed, even if a reliable regime were numerically under control, the correct asymptotic boundary conditions pertaining to branes are yet to be found. In contrast, for extremal branes one can exploit the fact that the near-horizon throat should be deep enough to ensure the reliability of the probe regime.

In order to further investigate the parametric control of our results, one can verify that the probe stack remains far away from the boundary of the interval for parametrically large times. To this end, one can consider rigid branes moving along $y$, starting from the initial conditions $y(0)=y_{0}, \dot{y}(0)=0$. The reduced Lagrangian

$$
\mathcal{L}_{\text {red }}=-T_{p} N_{p} V_{p 8} \sqrt{1-e^{2(B-A)} \dot{y}^{2}}
$$

leads to the conserved Hamiltonian

$$
H_{\mathrm{red}}=\frac{T_{p} N_{p} V_{p 8}}{\sqrt{1-e^{2(B-A)} \dot{y}^{2}}}=T_{p} N_{p} V_{p 8}\left(y_{0}\right)
$$

and thus, solving by quadrature, one finds that

$$
t=\int_{y_{0}}^{y} \frac{e^{B(u)-A(u)}}{\sqrt{1-\left(\frac{V_{p 8}(u)}{V_{p 8}\left(y_{0}\right)}\right)^{2}}} d u=\int_{y_{0}}^{y} \frac{g_{s}^{-\frac{3}{4}} e^{-\frac{u^{2}}{2}}}{u^{\frac{5}{9}} \sqrt{1-\left(\frac{u}{y_{0}}\right)^{\frac{4}{9}(p-2)} e^{\frac{p-5}{4}\left(u^{2}-y_{0}^{2}\right)}}}
$$

is indeed parametrically large in string units for $g_{s} \ll 1$. 


\section{Dudas-Mourad probe potentials}
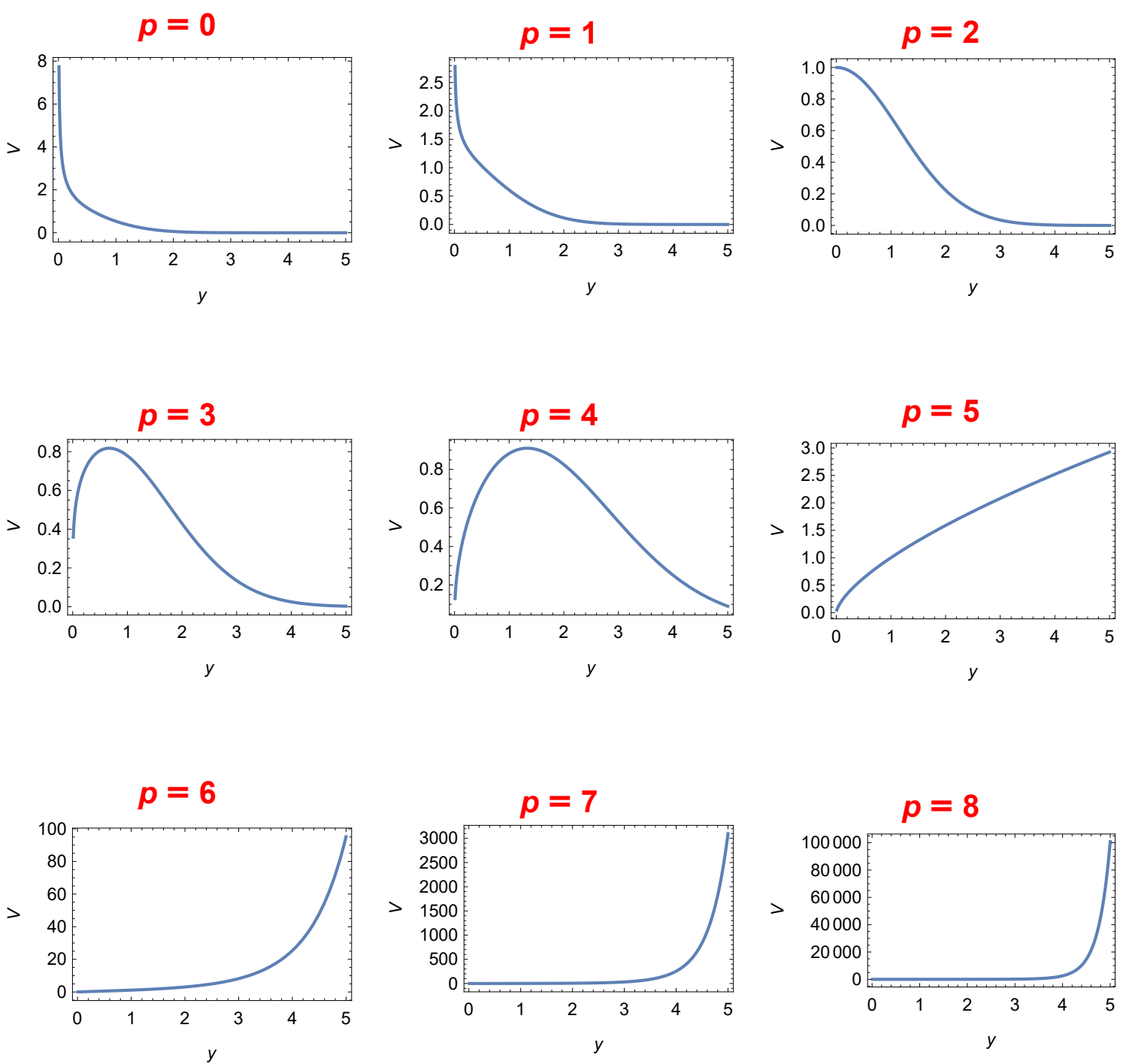

Figure 5. probe potentials for $g_{s}=1$ and $p \leq 8$. For $p<3$ the probe stack is repelled by the D8-branes, while for $p>4$ it is attracted to the D8-branes. A string amplitude computation yields a qualitatively similar behavior, despite the string-scale breaking of supersymmetry.

\subsubsection{Probe 8-branes in AdS $\times \mathbb{S}$ throats}

To conclude our analysis of probe branes, let us finally consider $N_{8}$ D8-branes probing the near-horizon geometries sourced by $N_{1} \gg N_{8}$ extremal D1-branes or $N_{3} \gg N_{8}$ extremal D3-branes in the orientifold models. For completeness we shall also consider 8-branes probing the $\mathrm{AdS}_{7} \times \mathbb{S}^{3}$ throat sourced by $N_{5} \gg N_{8}$ NS5-branes in the heterotic model. These comprise the last settings that we shall consider, since the 8-branes can wrap the internal spheres without collapsing in a vanishing cycle, while leaving enough dimensions to be parallel to the heavy stack. These are also the only cases where computations can 
Dudas-Mourad probe potentials
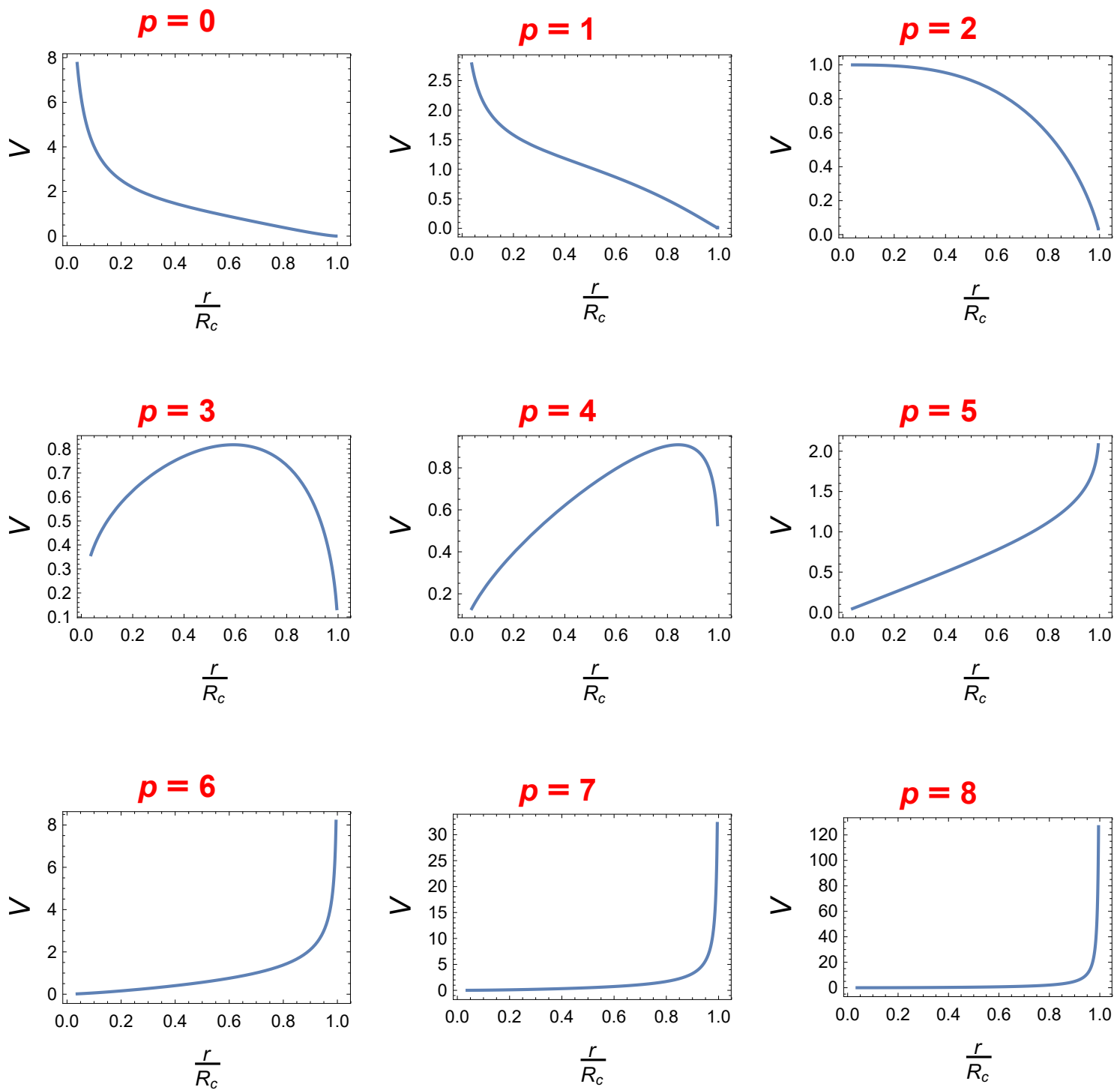

Figure 6. probe potentials for $g_{s}=1$ and $p \leq 8$, plotted as functions of the geodesic coordinate along the compact direction.

be compared to the results in the preceding sections, which hold in the opposite regime $N_{1}, N_{3}, N_{5} \ll N_{8}$. The respective potentials $V_{81}, V_{83}, V_{85}$ arise from the DBI contribution only, and take the form

$$
\begin{aligned}
& V_{81} \propto N_{8} T_{8} R^{7}\left(\frac{L}{Z}\right)^{2}, \\
& V_{85} \propto N_{8} T_{8} R^{3}\left(\frac{L}{Z}\right)^{6}
\end{aligned}
$$

for the $\operatorname{AdS} \times \mathbb{S}$ throats of eq. (3.8), up to an irrelevant (positive) constant, while

$$
V_{83} \sim \sqrt{2} \pi^{\frac{1}{4}} \alpha^{\prime \frac{9}{2}} g_{s}^{-\frac{3}{4}} N_{3}^{\frac{1}{4}} N_{8} T_{8} U^{4}\left(1+\frac{3}{8} g_{s} T \log \left(\frac{U}{u_{0}}\right)\right)
$$


in the near-horizon limit of the geometry of eq. (3.15) sourced by D3-branes in the type $0^{\prime} \mathrm{B}$ model. These potentials are attractive, which may at first glance appear in contradiction with the results in the following section. However, at large separation, 8-branes wrapped around the internal spheres should behave as (uncharged) 1-branes, 5-branes and 3-branes respectively, consistently with an attractive potential between branes of equal dimension.

\subsection{String-amplitude regime}

We can now compare the results of the preceding probe-brane analysis for uncharged branes to a string amplitude computation. The relevant leading-order amplitude encoding the interaction between parallel stack of $N_{p} \mathrm{D} p$-branes and $N_{q}$ D $q$-branes, ${ }^{22}$ with $p<q$ for definiteness, corresponds to the annulus. The transverse-channel integrand in the present cases takes the form [20]

$$
\widetilde{\mathcal{A}}_{p q} \propto N_{p} N_{q}\left(V_{8-q+p} O_{q-p}-O_{8-q+p} V_{q-p}\right)
$$

up to a (positive) normalization, where the characters are evaluated at $\mathfrak{q}=e^{-2 \pi \ell}$. In suitable units for the transverse separation $r$, the potential $V_{p q}$ is then given by

$$
V_{p q} \propto-N_{p} N_{q} \int_{0}^{\infty} \frac{d \ell}{\ell^{\frac{9-q}{2}}} \frac{\widetilde{A}_{p q}}{\eta^{8-q+p}}\left(\frac{2 \eta}{\vartheta_{2}}\right)^{\frac{q-p}{2}} e^{-\frac{r^{2}}{\ell}} .
$$

For large $r$, the integral is dominated by the large- $\ell$ region. In this region eq. (4.28) asymptotes to $\mathfrak{q}^{-\frac{1}{3}} \tilde{A}_{p q}$, with

$$
\begin{aligned}
\tilde{A}_{p q} & \propto V_{8-q+p} O_{q-p}-O_{8-q+p} V_{q-p} \\
& \sim 2(4-q+p) \mathfrak{q}^{\frac{1}{3}}
\end{aligned}
$$

so that for $q<7$ one finds

$$
V_{p q} \propto(q-p-4) \frac{N_{p} N_{q}}{r^{7-q}} .
$$

This potential is repulsive for $p<q-4$ and attractive for $p>q-4$. While the integral in eq. (4.29) diverges for $q \geq 7$, a distributional computation for $q=7,8$ yields a finite force stemming from potentials proportional to $(p-3) \log (r)$ and $(p-4) r$ respectively. In order to compare these results to the probe-brane analysis of the preceding section, the relevant cases are thus $p=q$, which leads to an attractive potential, consistently with eqs. (4.26) and (4.27), and $q=8$, which leads to a potential proportional to $(p-4) r$. Therefore, the latter interaction is repulsive for $p<4$ and attractive for $p>4$, consistently with the results in the preceding section. Let us remark that, in the absence of (linear) supersymmetry, as well as a flat space-time background, the agreement between the two computations, performed in complementary regimes, seems quite non-trivial, and suggests a deeper principle akin to the WGC, or the repulsive force conjecture, for uncharged extended objects.

\footnotetext{
${ }^{22}$ One can expect that an amplitude computation be reliable for $N_{p}, N_{q}=\mathcal{O}(1)$, in contrast to the probe regimes $N_{p} \gg N_{q}$ and $N_{p} \ll N_{q}$.
} 


\subsection{Holographic regime}

The appearance of (unstable) AdS geometries in the models that we have investigated, together with their connection to non-supersymmetric brane configurations, suggests that the world-volume gauge theories living on the branes could encode their dynamics holographically, including gravitational instabilities. Indeed, the perturbative instabilities studied in $[49,83]$ ought to correspond to operators with complex anomalous dimension $[84,85]$, while the holographic description of non-perturbative instabilities is more subtle [86-95] and has been proposed to be captured by dual RG flows $[96,97]$. In the latter picture, the putative CFT deformations ought to be "heavy", since their effect is suppressed in the large-flux limit, but some properties of brane dynamics can be understood holographically, if qualitatively, in the weak-coupling regime of the gauge theory.

Concretely, the weak-gravity repulsive effect that we have discussed in section 4.1 suggests that the gauge group undergoes a dynamical breaking according to [69, 98]

$$
\begin{aligned}
\mathrm{U}(N) & \rightarrow \mathrm{U}(N-\delta N) \times \mathrm{U}(\delta N), \\
\mathrm{USp}(2 N) & \rightarrow \mathrm{USp}(2 N-2 \delta N) \times \mathrm{USp}(2 \delta N)
\end{aligned}
$$

in the type $0^{\prime} \mathrm{B}$ model and the Sugimoto model respectively. However, the initial expectation value attained by scalars upon separating the branes would increase due to the repulsive force, and thus a conventional Higgs mechanism would not appear to be involved, at least not in its usual guise. While the bulk AdS geometry is expected to be dual to the IR regime of the world-volume gauge theory, which as we shall see is strongly coupled, one can make progress studying its weakly coupled UV counterpart, under the assumption that the qualitative character of brane interactions be preserved under RG flow. In the Sugimoto model, the relevant gauge theory arises projecting a supersymmetric one. In settings of this type the projected theory retains some properties of the parent theory [99-106], and we intend to explore this intriguing idea in future work. However, for the time being we shall focus on the weakly coupled UV regime of this gauge theory, alongside its $\mathrm{U}(N)$ counterpart in the type $0^{\prime} \mathrm{B}$ model. ${ }^{23}$

To begin with, one is to address the issue of which background the branes are placed in. According to general considerations along the lines of [79], one ought to place the branes in the flux-less limit of the back-reacted geometry of eq. (3.8). However, in the absence of supersymmetry the resulting configuration appears highly curved and far outside the EFT regime of validity, and in particular there is no Minkowski solution to replace it. However, introducing $N_{8} \gg 1$ D8-branes sourcing the static Dudas-Mourad geometry of eq. (3.12), the situation appears more tame, since $N \gg N_{8}$ D1-branes placed in the controlled region described in section 4.1.2 would dominate the back-reaction. If this construction is reliable, standard decoupling arguments [79] should lead to a two-dimensional world-volume gauge theory on flat space-time. In the case of the Sugimoto model, the massless perturbative spectrum has been described in [5], while the case of the type $0^{\prime} \mathrm{B}$ model was studied in [47].

\footnotetext{
${ }^{23}$ For similar considerations on D3-branes in the type $0^{\prime} \mathrm{B}$ model, for which the gauge theory is strongly coupled in the UV, see [47].
} 
The corresponding world-volume (Euclidean) effective action $S_{\mathrm{D} 1}$ then takes the schematic form

$$
\begin{aligned}
S_{\mathrm{D} 1}^{E}=\frac{1}{g_{\mathrm{YM}}^{2}} \operatorname{Tr} & \int d^{2} \zeta\left(\left(\partial_{+} A_{-}\right)^{2}+\partial_{+} X_{i}\left[\mathcal{D}_{-}, X_{i}\right]-\frac{1}{4}\left[X_{i}, X_{j}\right]\left[X_{i}, X_{j}\right]\right. \\
& \left.+\psi_{+}\left[\mathcal{D}_{-}, \psi_{+}\right]+\psi_{-} \partial_{+} \psi_{-}+\psi_{-} \Gamma_{i}\left[X_{i}, \psi_{+}\right]+\lambda_{-}^{A} \partial_{+} \lambda_{-}^{A}\right)
\end{aligned}
$$

in the (Euclidean) light-cone gauge $A_{+}=0$. In the Sugimoto model, in contrast to its supersymmetric counterpart, the scalars $X_{i}$ which comprise a vector of the transverse rotation group $\mathrm{SO}(8)$ are in the anti-symmetric representation of $\operatorname{USp}(2 N)$, while the adjoint is symmetric and the world-volume fermion $\psi_{+}$(resp. $\psi_{-}$) is in the symmetric (resp. anti-symmetric) representation and is a $\mathrm{SO}(8)$ spinor. The $\lambda_{-}^{A}$ are bifundamental fermions of $\operatorname{USp}(2 N) \times \operatorname{USp}\left(2 N_{f}\right)$ with $N_{f}=16$ "flavors", and arise from (massless modes of) open strings stretching between the D1-branes and the $\overline{\mathrm{D} 9}$-branes. The type $0^{\prime} \mathrm{B}$ model is analogous mutatis mutandis, with the important difference that the scalars are in the adjoint representation of the gauge group $\mathrm{U}(N)$, since the Möbius strip contribution does not modify the structure encoded in the annulus due to the vanishing O9-plane tension. While the light-cone gauge is convenient, since in two dimensions ghosts decouple [107] and the gauge field can be integrated out exactly, ${ }^{24}$ here we shall content ourselves with a one-loop analysis. In this respect, the $\beta$ function of the gauge coupling depends only on the (perturbative) matter content. In order to derive it, let us recall that, in four dimensions, the result

$$
\beta_{4 d}=b_{1} \frac{g_{\mathrm{YM}}^{3}}{16 \pi^{2}}
$$

arises from the (dimension-independent) $a_{4}$ coefficient in the heat-kernel expansion of the one-loop functional determinant [109]. Therefore, in the two-dimensional case the bare coupling $g_{0}$ would be related to the renormalized coupling according to

$$
\frac{1}{g_{0}^{2}}=\frac{1}{g_{\mathrm{YM}}^{2}(\mu)}-\frac{b_{1}}{4 \pi} \frac{1}{\mu^{2}}
$$

In terms of the dimensionless coupling $g_{\mathrm{YM}} \equiv \widehat{g} \mu$, the one-loop $\beta$ function is then

$$
\widehat{\beta}_{2 d}=-\widehat{g}+\frac{b_{1}}{4 \pi} \widehat{g}^{3}
$$

with $[110-112]$

$$
b_{1}^{\mathrm{USp}(2 N)}=\frac{9 N+N_{f}-15}{3}, \quad b_{1}^{\mathrm{U}(N)}=\frac{9 N+2 N_{f}}{3}
$$

for the Sugimoto model and the type $0^{\prime} \mathrm{B}$ model respectively. Therefore, the gauge coupling eventually flows to a strongly coupled region, which could exhibit confinement or screening [113], even in the large- $N$ limit where the suitable parameter is the 't Hooft coupling. From the perspective of the bulk, the IR behavior of the gauge coupling ought to reflect the

\footnotetext{
${ }^{24}$ Non-perturbative methods of this type in light-cone gauge have been recently employed in [108].
} 
radial perturbations of the dilaton described in [13]. Specifically, power-like perturbations $\phi-\phi_{\text {AdS }} \propto|r|^{-\lambda}$ about the fixed-point AdS throat, encoded in eqs. (3.1) and (3.10), would translate into the IR $\beta$ function

$$
\widehat{\beta}_{\mathrm{IR}}=(2 \lambda-1)\left(\widehat{g}-\widehat{g}^{*}\right),
$$

since $r$ is related to the Poincaré-patch holographic coordinate $z \mapsto \frac{1}{\mu}$ according to $|r|=\frac{z^{2}}{2}$. In particular, the two independent power-like perturbations that decay in the IR yield [13]

$$
\widehat{\beta}_{\mathrm{IR}}=\sqrt{5}\left(\widehat{g}-\widehat{g}^{*}\right) \quad \text { or } \quad \widehat{\beta}_{\mathrm{IR}}=\sqrt{13}\left(\widehat{g}-\widehat{g}^{*}\right)
$$

in the orientifold models. The overall picture appears daunting, but the (non-local) quartic effective action obtained integrating out the gauge field is potentially amenable to large- $N$ Hubbard-Stratonovich techniques [114, 115] or non-Abelian bosonization [116].

As we have anticipated, one can still attempt to make some progress studying the UV regime computing the one-loop effective potential. To this end, in order to connect with a space-time interpretation, we shall consider "geometric" configurations that describe two parallel stacks indexed by $\alpha=1,2$ and transverse positions $\mathbf{x}^{(\alpha)}$. The corresponding configuration for the scalars $X_{k}$ is

$$
X_{k}=\frac{g_{\mathrm{YM}}}{\sqrt{2 N}} \sum_{\alpha=1}^{2} \Omega_{\alpha} x_{k}^{(\alpha)},
$$

where the $\Omega_{\alpha}$ are block-diagonal. In detail, they are the projections onto the first and second stacks of the (symplectic-)trace singlet, which is the symplectic matrix $i \Omega$ in the Sugimoto model and the identity matrix in the type $0^{\prime} \mathrm{B}$ model. The resulting quadratic kinetic operator yields a functional determinant akin to that studied in [17, 18], and using a covariant, Feynman-like gauge the contributions of the gauge field cancels that of the ghosts. This is to be expected on account of our preceding considerations. Furthermore, the fermionic terms also vanish in the Sugimoto model, while in the type $0^{\prime} \mathrm{B}$ they cancel the bosonic contribution, mirroring the results of [18] for self-dual D3-branes. All in all, for the Sugimoto model the quadratic action for fluctuations $\delta X_{i} \equiv \delta X_{i}^{a} t_{a}$, decomposed in an orthogonal basis $\left\{t_{a}\right\}$ of the space of (imaginary) anti-symmetric matrices, takes the form

$$
S_{\mathrm{D} 1}^{(2)}=\int d^{2} \zeta\left(\partial_{+} \delta X_{i}^{a} \partial_{-} \delta X_{i}^{a}+\frac{1}{2} \delta X_{i}^{a}\left(M^{2}\right)_{i j}^{a b} \delta X_{j}^{b}\right)
$$

where the (positive semidefinite) mass matrix

$$
\left(M^{2}\right)_{i j}^{a b}=\frac{g_{\mathrm{YM}}^{2}}{N} \delta_{i j} \sum_{\alpha, \beta} \mathbf{x}^{(\alpha)} \cdot \mathbf{x}^{(\beta)} \omega_{a b}^{(\alpha \beta)}, \quad \omega_{a b}^{(\alpha \beta)} \equiv \operatorname{Tr}\left(\left[\Omega_{\alpha}, t_{a}\right]\left[\Omega_{\beta}, t_{b}\right]\right)
$$

arises from the quartic potential of eq. (4.33), and one is thus led to the one-loop effective potential

$$
V_{\mathrm{D} 1}^{(1)}=\frac{1}{2} \operatorname{Tr} \int \frac{d^{2} p}{(2 \pi)^{2}} \log \left(p^{2}+M^{2}\right)
$$


The non-vanishing eigenvalues of the mass matrix comprise not only the separation $r^{2} \equiv$ $\left|\mathbf{x}^{(1)}-\mathbf{x}^{(2)}\right|^{2}$ between the stacks, as in the case of orthogonal and unitary gauge groups, but also (twice) the mean position $\left|\mathbf{x}^{(1)}+\mathbf{x}^{(2)}\right|^{2}$. Furthermore, when a stack contains more than one brane, there are eigenvalues proportional to the square $\left|\mathbf{x}^{(\alpha)}\right|^{2}$ of its position. While we were not able to provide a complete explanation of this behaviour, which is due to the non-Abelian nature of the (projected) symplectic matrices $\Omega_{\alpha}$ and thus reminiscent of brane polarization [117], when interpreted in terms of smooth space-time geometry the apparent breaking of translational symmetry could presumably be ascribed to the absence of a flat background in which the branes can be placed, along with the presence of a force acting on them. This resonates with our preceding considerations, whereby the world-volume gauge theory can be constructed placing probe branes in the controlled region of the Dudas-Mourad geometry: the force acting on probes, which we have discussed in section 4.1.2, appears to be encoded in the one-loop effective potential of the world-volume gauge theory, at least for the Sugimoto model.

Focusing on the contribution to the trace in eq. (4.43) that depends on the brane separation $r$, one finds

$$
\left.V_{\mathrm{D} 1}^{(1)}\right|_{\mathrm{sep}}=\frac{m}{\pi} \int_{0}^{\Lambda_{\mathrm{UV}}^{2}} d s \log \left(s+\frac{g_{\mathrm{YM}}^{2} r^{2}}{N}\right)
$$

with $m$ the corresponding multiplicity. Notice that the renormalized dimensionless coupling $\widehat{g}\left(\mu_{\text {sep }}\right)$ evaluated at the separation scale $\mu_{\text {sep }}^{-1} \equiv r / \Lambda_{\mathrm{UV}}$ appears, and the perturbative regime translates into the requirement $\widehat{g}\left(\mu_{\text {sep }}\right) \ll 1$, so that

$$
\left.V_{\mathrm{D} 1}^{(1)}\right|_{\mathrm{sep}} \sim-\frac{m}{\pi} \frac{g_{\mathrm{YM}}^{2} r^{2}}{N} \log \left(\frac{g_{\mathrm{YM}}^{2} r^{2}}{N \Lambda_{\mathrm{UV}}^{2}}\right)
$$

indeed exhibits a repulsive behavior induced by tunneling. This result, although marginally reliable at the tunneling scale, appears in agreement with axial-gauge computations, and is consistent with our preceding considerations. Moreover, as expected, for $N=1$ the (gauge singlet) scalars decouple, and thus their effective potential receives no corrections even beyond the one-loop level. This is reflected in eq. (4.45) by the fact that $m=0$ for $N=1$.

\section{Conclusions}

In this paper we have investigated the interactions of branes in non-supersymmetric string models. In particular, we have focused on the $\mathrm{USp}(32)[5]$ and $\mathrm{U}(32)$ "type $0^{\prime} \mathrm{B}$ " $[3,4]$ orientifold models and on the $\mathrm{SO}(16) \times \mathrm{SO}(16)$ heterotic model of $[1,2]$, computing static interaction potentials for parallel stack of branes. Despite the absence of supersymmetry, the presence of R-R charges entails the existence of charged extremal branes in the spectrum, which comprise D1 and D5-branes in the Sugimoto model and D1, D3, D5 and D7-branes in the type $0^{\prime} \mathrm{B}$ model. In addition, we have included heterotic NS5-branes in our analysis, since their behaviour mirrors that of D1-branes to a certain extent. On the other hand, we have excluded D5 - D5 and D7 - D7 interactions, since the corresponding background 
geometries and string amplitudes present a number of subtleties. Our computations span a variety of regimes: whenever one stack is parametrically heavier than the other, one can replace the heavy stack with the corresponding background geometry probed by the light stack, while whenever both stacks are parametrically light one can compute the interaction potential via the annulus amplitude, provided that the two stacks do not share the same charges. Finally, we have investigated the world-volume gauge theory of D1-branes in the Sugimoto model in an attempt to extend our results beyond the perturbative regime, connecting them to a top-down non-supersymmetric holography of the type discussed in $[13,96]$. We have computed the one-loop $\beta$ function and effective potential for separated branes, finding a departure from the supersymmetric case and from the type $0^{\prime} \mathrm{B}$ model.

The interaction potentials that we have obtained are qualitatively consistent among these complementary regimes whenever comparisons are possible. Namely, separated stacks either attract or repel in each case. The agreement is non-trivial, since there is no apparent principle that protects this behavior upon increase of the strength of gravitational backreaction. Moreover, for branes that share the same charge(s), we found a novel mechanism that realizes the Weak Gravity Conjecture via a renormalization of the effective charge-to-tension ratio. This peculiar effect arises from an interaction between the branes mediated by the supersymmetry-breaking ingredients, which at the level of the ten-dimensional EFT are reflected by the gravitational tadpole potential in eq. (2.4). This behavior is also consistent with the world-volume computations that we have presented in section 4.3, although its reliability is confined to the UV due to asymptotic freedom. On the other hand, a proper holographic comparison would entail flowing to the strongly coupled IR, where arguments from the bulk suggest that a large- $N$ fixed point ought to exist. We would like to pursue this direction further in future work, possibly relying on non-perturbative large- $N$ and two-dimensional methods.

All in all, our findings suggest that the instructive lessons that have been gathered from brane dynamics can be robust, to some extent, with respect to the dramatic effects of high-energy supersymmetry breaking. One can expect that deeper connections with holography and Swampland proposals can be unveiled from this perspective, which would nicely complement the bottom-up considerations of $[118,119]$ (see also $[39,40]$ ). In this paper we have only taken a first step toward a deeper understanding of these fundamental issues, and a more detailed investigation could encompass D5 - D5 and D7 - D7 interactions, either in the probe regime or in the string-amplitude regime, and more generally probe-brane potentials for the (so far elusive) background geometries sourced by D5 and D7-branes. Other avenues for future research include exploring further the back-reaction and thermodynamics of non-extremal branes, as well as involving higher-order Wess-Zumino terms along the lines of [120] to take into account the effects of R-R interactions for branes of different dimensions. Aside from cases where the leading contribution vanishes, one can expect that couplings of this type be required to all orders, at least in $\alpha^{\prime}$, in order to circumvent a Dine-Seiberg-like argument [121]. Some progress along these lines has been achieved via all-order string-amplitude computations (see, e.g., [122, 123]) and via T-duality in the context of cosmology [124-133]. Furthermore, from the perspective of holography, it would be interesting to understand whether the IR fixed-point structure exhibited by the 
near-horizon AdS throats discussed in section 3 can be reproduced via strong-coupling effects in the putative dual gauge theory. While the limit of a large number of branes appears necessary in order to probe this regime, small- $N$ computations seem to reveal intriguing hints concerning the scenarios proposed in $[13,96]$ and their connections to the conjectures put forth in $[57,61,134-138]$. These and other related issues are currently under investigation.

\section{Acknowledgments}

It is a pleasure to thank C. Angelantonj, E. Dudas, M. Bianchi and A. Sagnotti for helpful feedback during the development of this work. I am also grateful to G. Bogna, S. Raucci, S. Bottaro, D. Bufalini, S. Lanza and A. Faraggi for insightful discussions and feedback on the manuscript.

This work was supported by the Fonds de la Recherche Scientifique - FNRS under Grants No. F.4503.20 ("HighSpinSymm") and T.0022.19 ("Fundamental issues in extended gravitational theories").

Open Access. This article is distributed under the terms of the Creative Commons Attribution License (CC-BY 4.0), which permits any use, distribution and reproduction in any medium, provided the original author(s) and source are credited.

\section{References}

[1] L. Álvarez-Gaumé, P.H. Ginsparg, G.W. Moore and C. Vafa, An $O(16) \times O(16)$ Heterotic String, Phys. Lett. B 171 (1986) 155 [INSPIRE].

[2] L.J. Dixon and J.A. Harvey, String Theories in Ten-Dimensions Without Space-Time Supersymmetry, Nucl. Phys. B 274 (1986) 93 [InSPIRE].

[3] A. Sagnotti, Some properties of open string theories, in International Workshop on Supersymmetry and Unification of Fundamental Interactions (SUSY 95), (1995), pp. 473-484 [hep-th/9509080] [INSPIRE].

[4] A. Sagnotti, Surprises in open string perturbation theory, Nucl. Phys. B Proc. Suppl. 56 (1997) 332 [hep-th/9702093] [INSPIRE].

[5] S. Sugimoto, Anomaly cancellations in type-I D9- $\bar{D} 9$ system and the USp(32) string theory, Prog. Theor. Phys. 102 (1999) 685 [hep-th/9905159] [INSPIRE].

[6] I. Antoniadis, E. Dudas and A. Sagnotti, Brane supersymmetry breaking, Phys. Lett. B 464 (1999) 38 [hep-th/9908023] [INSPIRE].

[7] C. Angelantonj, Comments on open string orbifolds with a nonvanishing B(ab), Nucl. Phys. B 566 (2000) 126 [hep-th/9908064] [INSPIRE].

[8] G. Aldazabal and A.M. Uranga, Tachyon free nonsupersymmetric type IIB orientifolds via Brane - anti-brane systems, JHEP 10 (1999) 024 [hep-th/9908072] [INSPIRE].

[9] C. Angelantonj, I. Antoniadis, G. D'Appollonio, E. Dudas and A. Sagnotti, Type I vacua with brane supersymmetry breaking, Nucl. Phys. B $\mathbf{5 7 2}$ (2000) 36 [hep-th/9911081] [INSPIRE]. 
[10] T. Coudarchet, E. Dudas and H. Partouche, Geometry of orientifold vacua and supersymmetry breaking, arXiv:2105.06913 [INSPIRE].

[11] N. Cribiori, D. Lüst and M. Scalisi, The gravitino and the swampland, JHEP 06 (2021) 071 [arXiv:2104.08288] [INSPIRE].

[12] A. Castellano, A. Font, A. Herraez and L.E. Ibáñez, A Gravitino Distance Conjecture, arXiv:2104.10181 [INSPIRE].

[13] R. Antonelli and I. Basile, Brane annihilation in non-supersymmetric strings, JHEP 11 (2019) 021 [arXiv: 1908.04352] [INSPIRE].

[14] I. Basile and S. Lanza, de Sitter in non-supersymmetric string theories: no-go theorems and brane-worlds, JHEP 10 (2020) 108 [arXiv: 2007.13757] [INSPIRE].

[15] C. Angelantonj and A. Armoni, Nontachyonic type OB orientifolds, nonsupersymmetric gauge theories and cosmological RG flow, Nucl. Phys. B 578 (2000) 239 [hep-th/9912257] [INSPIRE].

[16] C. Angelantonj and A. Armoni, RG flow, Wilson loops and the dilaton tadpole, Phys. Lett. B 482 (2000) 329 [hep-th/0003050] [INSPIRE].

[17] K. Zarembo, Coleman-Weinberg mechanism and interaction of D3-branes in type 0 string theory, Phys. Lett. B 462 (1999) 70 [hep-th/9901106] [INSPIRE].

[18] A.A. Tseytlin and K. Zarembo, Effective potential in nonsupersymmetric $\mathrm{SU}(N) \times \mathrm{SU}(N)$ gauge theory and interactions of type 0 D3-branes, Phys. Lett. B 457 (1999) 77 [hep-th/9902095] [INSPIRE].

[19] N. Arkani-Hamed, L. Motl, A. Nicolis and C. Vafa, The string landscape, black holes and gravity as the weakest force, JHEP 06 (2007) 060 [hep-th/0601001] [INSPIRE].

[20] E. Dudas, J. Mourad and A. Sagnotti, Charged and uncharged D-branes in various string theories, Nucl. Phys. B 620 (2002) 109 [hep-th/0107081] [INSPIRE].

[21] J.D. Blum and K.R. Dienes, Duality without supersymmetry: The case of the $\mathrm{SO}(16) \times \mathrm{SO}(16)$ string, Phys. Lett. B 414 (1997) 260 [hep-th/9707148] [INSPIRE].

[22] J.D. Blum and K.R. Dienes, Strong/weak coupling duality relations for nonsupersymmetric string theories, Nucl. Phys. B 516 (1998) 83 [hep-th/9707160] [INSPIRE].

[23] P. Hořava and E. Witten, Heterotic and type-I string dynamics from eleven-dimensions, Nucl. Phys. B 460 (1996) 506 [hep-th/9510209] [INSPIRE].

[24] A.E. Faraggi and M. Tsulaia, On the Low Energy Spectra of the Nonsupersymmetric Heterotic String Theories, Eur. Phys. J. C 54 (2008) 495 [arXiv: 0706.1649] [InSPIRE].

[25] A. Sagnotti, Open Strings and their Symmetry Groups, in NATO Advanced Summer Institute on Nonperturbative Quantum Field Theory (Cargese Summer Institute), (1987) [hep-th/0208020] [INSPIRE].

[26] G. Pradisi and A. Sagnotti, Open String Orbifolds, Phys. Lett. B 216 (1989) 59 [InSPIRE].

[27] P. Hořava, Strings on World Sheet Orbifolds, Nucl. Phys. B 327 (1989) 461 [InSPIRE].

[28] P. Hořava, Background Duality of Open String Models, Phys. Lett. B 231 (1989) 251 [INSPIRE].

[29] M. Bianchi and A. Sagnotti, On the systematics of open string theories, Phys. Lett. B 247 (1990) 517 [INSPIRE]. 
[30] M. Bianchi and A. Sagnotti, Twist symmetry and open string Wilson lines, Nucl. Phys. B 361 (1991) 519 [INSPIRE].

[31] M. Bianchi, G. Pradisi and A. Sagnotti, Toroidal compactification and symmetry breaking in open string theories, Nucl. Phys. B 376 (1992) 365 [inSPIRE].

[32] A. Sagnotti, A note on the Green-Schwarz mechanism in open string theories, Phys. Lett. B 294 (1992) 196 [hep-th/9210127] [INSPIRE].

[33] E. Dudas, Theory and phenomenology of type-I strings and M-theory, Class. Quant. Grav. 17 (2000) R41 [hep-ph/0006190] [INSPIRE].

[34] C. Angelantonj and A. Sagnotti, Open strings, Phys. Rept. 371 (2002) 1 [Erratum ibid. 376 (2003) 407] [hep-th/0204089] [INSPIRE].

[35] J. Mourad and A. Sagnotti, An Update on Brane Supersymmetry Breaking, arXiv:1711.11494 [INSPIRE].

[36] J. Mourad and A. Sagnotti, String (In)Stability Issues with Broken Supersymmetry, arXiv:2107.04064 [INSPIRE].

[37] E. Dudas and J. Mourad, Consistent gravitino couplings in nonsupersymmetric strings, Phys. Lett. B 514 (2001) 173 [hep-th/0012071] [INSPIRE].

[38] G. Pradisi and F. Riccioni, Geometric couplings and brane supersymmetry breaking, Nucl. Phys. B 615 (2001) 33 [hep-th/0107090] [INSPIRE].

[39] G. Buratti, M. Delgado and A.M. Uranga, Dynamical tadpoles, stringy cobordism, and the SM from spontaneous compactification, JHEP 06 (2021) 170 [arXiv:2104.02091] [INSPIRE].

[40] G. Buratti, J. Calderón-Infante, M. Delgado and A.M. Uranga, Dynamical Cobordism and Swampland Distance Conjectures, JHEP 10 (2021) 037 [arXiv:2107.09098] [INSPIRE].

[41] K.R. Dienes, New string partition functions with vanishing cosmological constant, Phys. Rev. Lett. 65 (1990) 1979 [inSPIRE].

[42] K.R. Dienes, Generalized Atkin-Lehner Symmetry, Phys. Rev. D 42 (1990) 2004 [InSPIRE].

[43] S. Kachru, J. Kumar and E. Silverstein, Vacuum energy cancellation in a nonsupersymmetric string, Phys. Rev. D 59 (1999) 106004 [hep-th/9807076] [INSPIRE].

[44] C. Angelantonj and M. Cardella, Vanishing perturbative vacuum energy in nonsupersymmetric orientifolds, Phys. Lett. B 595 (2004) 505 [hep-th/0403107] [InSPIRE].

[45] S. Abel, K.R. Dienes and E. Mavroudi, Towards a nonsupersymmetric string phenomenology, Phys. Rev. D 91 (2015) 126014 [arXiv:1502.03087] [InSPIRE].

[46] S. Abel and R.J. Stewart, Exponential suppression of the cosmological constant in nonsupersymmetric string vacua at two loops and beyond, Phys. Rev. D 96 (2017) 106013 [arXiv: 1701.06629] [INSPIRE].

[47] E. Dudas and J. Mourad, D-branes in nontachyonic OB orientifolds, Nucl. Phys. B 598 (2001) 189 [hep-th/0010179] [INSPIRE].

[48] J. Mourad and A. Sagnotti, AdS Vacua from Dilaton Tadpoles and Form Fluxes, Phys. Lett. $B \mathbf{7 6 8}$ (2017) 92 [arXiv:1612.08566] [INSPIRE].

[49] I. Basile, J. Mourad and A. Sagnotti, On Classical Stability with Broken Supersymmetry, JHEP 01 (2019) 174 [arXiv:1811.11448] [InSPIRE]. 
[50] J.M. Maldacena and H. Ooguri, Strings in $A d S_{3}$ and $\mathrm{SL}(2, \mathbb{R})$ WZW model 1.: The Spectrum, J. Math. Phys. 42 (2001) 2929 [hep-th/0001053] [InSPIRE].

[51] E. Dudas and J. Mourad, Brane solutions in strings with broken supersymmetry and dilaton tadpoles, Phys. Lett. B 486 (2000) 172 [hep-th/0004165] [inSPIRE].

[52] G.T. Horowitz and A. Strominger, Black strings and P-branes, Nucl. Phys. B 360 (1991) 197 [INSPIRE].

[53] K.S. Stelle, BPS branes in supergravity, in ICTP Summer School in High-energy Physics and Cosmology, (1998) [hep-th/9803116] [INSPIRE].

[54] I.R. Klebanov and A.A. Tseytlin, D-branes and dual gauge theories in type 0 strings, Nucl. Phys. B 546 (1999) 155 [hep-th/9811035] [INSPIRE].

[55] I. Basile, Supersymmetry Breaking and Stability in String Vacua: brane dynamics, bubbles and the swampland, Riv. Nuovo Cim. 1 (2021) 98 [arXiv:2107.02814] [INSPIRE].

[56] R. Antonelli, Black Objects without a Vacuum, Ph.D. thesis, Pisa, Scuola Normale Superiore, Italy (2020).

[57] D. Lüst, E. Palti and C. Vafa, AdS and the Swampland, Phys. Lett. B 797 (2019) 134867 [arXiv: 1906. 05225] [INSPIRE].

[58] D. Lüst and D. Tsimpis, AdS 2 type-IIA solutions and scale separation, JHEP 07 (2020) 060 [arXiv: 2004.07582] [INSPIRE].

[59] G.B. De Luca and A. Tomasiello, Leaps and bounds towards scale separation, arXiv:2104.12773 [INSPIRE].

[60] H. Ooguri and C. Vafa, Non-supersymmetric AdS and the Swampland, Adv. Theor. Math. Phys. 21 (2017) 1787 [arXiv:1610.01533] [INSPIRE].

[61] H. Ooguri, E. Palti, G. Shiu and C. Vafa, Distance and de Sitter Conjectures on the Swampland, Phys. Lett. B $\mathbf{7 8 8}$ (2019) 180 [arXiv:1810.05506] [INSPIRE].

[62] G. Obied, H. Ooguri, L. Spodyneiko and C. Vafa, de Sitter Space and the Swampland, arXiv: 1806.08362 [INSPIRE].

[63] S.K. Garg and C. Krishnan, Bounds on Slow Roll and the de Sitter Swampland, JHEP 11 (2019) 075 [arXiv:1807.05193] [INSPIRE].

[64] H.J. Boonstra, K. Skenderis and P.K. Townsend, The domain wall/QFT correspondence, JHEP 01 (1999) 003 [hep-th/9807137] [INSPIRE].

[65] O. Aharony, S.S. Gubser, J.M. Maldacena, H. Ooguri and Y. Oz, Large N field theories, string theory and gravity, Phys. Rept. 323 (2000) 183 [hep-th/9905111] [INSPIRE].

[66] J.D. Brown and C. Teitelboim, Dynamical Neutralization of the Cosmological Constant, Phys. Lett. B 195 (1987) 177 [INSPIRE].

[67] J.D. Brown and C. Teitelboim, Neutralization of the Cosmological Constant by Membrane Creation, Nucl. Phys. B 297 (1988) 787 [InSPIRE].

[68] J.M. Maldacena, J. Michelson and A. Strominger, Anti-de Sitter fragmentation, JHEP 02 (1999) 011 [hep-th/9812073] [INSPIRE].

[69] N. Seiberg and E. Witten, The D1/D5 system and singular CFT, JHEP 04 (1999) 017 [hep-th/9903224] [INSPIRE]. 
[70] J.J. Blanco-Pillado, D. Schwartz-Perlov and A. Vilenkin, Quantum Tunneling in Flux Compactifications, JCAP 12 (2009) 006 [arXiv:0904.3106] [INSPIRE].

[71] A.R. Brown and A. Dahlen, Small Steps and Giant Leaps in the Landscape, Phys. Rev. D 82 (2010) 083519 [arXiv: 1004.3994] [INSPIRE].

[72] A.R. Brown and A. Dahlen, Giant Leaps and Minimal Branes in Multi-Dimensional Flux Landscapes, Phys. Rev. D 84 (2011) 023513 [arXiv:1010.5241] [inSPIRE].

[73] E.A. Bergshoeff, M. de Roo, S.F. Kerstan and F. Riccioni, IIB supergravity revisited, JHEP 08 (2005) 098 [hep-th/0506013] [INSPIRE].

[74] E.A. Bergshoeff, M. de Roo, S.F. Kerstan, T. Ortín and F. Riccioni, SL(2, R)-invariant IIB Brane Actions, JHEP 02 (2007) 007 [hep-th/0611036] [INSPIRE].

[75] E.A. Bergshoeff and F. Riccioni, String Solitons and T-duality, JHEP 05 (2011) 131 [arXiv: 1102.0934] [INSPIRE].

[76] E.A. Bergshoeff and F. Riccioni, Heterotic wrapping rules, JHEP 01 (2013) 005 [arXiv: 1210.1422] [INSPIRE].

[77] E.A. Bergshoeff, V.A. Penas, F. Riccioni and S. Risoli, Non-geometric fluxes and mixed-symmetry potentials, JHEP 11 (2015) 020 [arXiv: 1508.00780] [INSPIRE].

[78] R. Blumenhagen and A. Font, Dilaton tadpoles, warped geometries and large extra dimensions for nonsupersymmetric strings, Nucl. Phys. B 599 (2001) 241 [hep-th/0011269] [InSPIRE].

[79] J.M. Maldacena, The large $N$ limit of superconformal field theories and supergravity, Int. J. Theor. Phys. 38 (1999) 1113 Adv. Theor. Math. Phys. 2 (1998) 231 [hep-th/9711200] [INSPIRE].

[80] M. Bianchi and A. Sagnotti, The Partition Function of the $\mathrm{SO}(8192)$ Bosonic String, Phys. Lett. B 211 (1988) 407 [InSPIRE].

[81] S.R. Coleman, The Fate of the False Vacuum. 1. Semiclassical Theory, Phys. Rev. D 15 (1977) 2929 [Erratum ibid. 16 (1977) 1248] [INSPIRE].

[82] C.G. Callan Jr. and S.R. Coleman, The Fate of the False Vacuum. 2. First Quantum Corrections, Phys. Rev. D 16 (1977) 1762 [INSPIRE].

[83] S.S. Gubser and I. Mitra, Some interesting violations of the Breitenlohner-Freedman bound, JHEP 07 (2002) 044 [hep-th/0108239] [INSPIRE].

[84] I.R. Klebanov, Tachyon stabilization in the AdS/CFT correspondence, Phys. Lett. B 466 (1999) 166 [hep-th/9906220] [INSPIRE].

[85] O. DeWolfe, D.Z. Freedman, S.S. Gubser, G.T. Horowitz and I. Mitra, Stability of AdS(p) x M(q) compactifications without supersymmetry, Phys. Rev. D 65 (2002) 064033 [hep-th/0105047] [INSPIRE].

[86] S. de Haro, I. Papadimitriou and A.C. Petkou, Conformally Coupled Scalars, Instantons and Vacuum Instability in AdS $S_{4}$, Phys. Rev. Lett. 98 (2007) 231601 [hep-th/0611315] [INSPIRE].

[87] I. Papadimitriou, Multi-Trace Deformations in AdS/CFT: Exploring the Vacuum Structure of the Deformed CFT, JHEP 05 (2007) 075 [hep-th/0703152] [INSPIRE].

[88] J. Maldacena, Vacuum decay into Anti de Sitter space, arXiv:1012.0274 [INSPIRE].

[89] J.L.F. Barbón and E. Rabinovici, Holography of AdS vacuum bubbles, JHEP 04 (2010) 123 [arXiv: 1003.4966] [INSPIRE]. 
[90] J.L.F. Barbón and E. Rabinovici, Holography of AdS vacuum bubbles, Nucl. Phys. B Proc. Suppl. 216 (2011) 121 [INSPIRE].

[91] D. Harlow, Metastability in Anti de Sitter Space, arXiv:1003.5909 [InSPIRE].

[92] T. Maxfield and S. Sethi, Domain Walls, Triples and Acceleration, JHEP 08 (2014) 066 [arXiv: 1404.2564] [INSPIRE].

[93] A. Bhattacharyya, L.-Y. Hung and C.M. Melby-Thompson, Instantons and Entanglement Entropy, JHEP 10 (2017) 081 [arXiv:1703.01611] [INSPIRE].

[94] P. Burda, R. Gregory and A. Jain, Holographic reconstruction of bubble spacetimes, Phys. Rev. D 99 (2019) 026003 [arXiv: 1804.05202] [InSPIRE].

[95] S. Hirano, Brown-Teitelboim instantons and thermodynamics of de Sitter and anti-de Sitter space, Phys. Rev. D 98 (2018) 046020 [arXiv: 1804.09985] [InSPIRE].

[96] R. Antonelli, I. Basile and A. Bombini, AdS Vacuum Bubbles, Holography and Dual RG Flows, Class. Quant. Grav. 36 (2019) 045004 [arXiv: 1806. 02289] [INSPIRE].

[97] J.K. Ghosh, E. Kiritsis, F. Nitti and L.T. Witkowski, Revisiting Coleman-de Luccia transitions in the AdS regime using holography, JHEP 09 (2021) 065 [arXiv:2102.11881] [INSPIRE].

[98] E. Witten, Baryons and branes in anti-de Sitter space, JHEP 07 (1998) 006 [hep-th/9805112] [INSPIRE].

[99] S. Kachru and E. Silverstein, 4-D conformal theories and strings on orbifolds, Phys. Rev. Lett. 80 (1998) 4855 [hep-th/9802183] [INSPIRE].

[100] A.E. Lawrence, N. Nekrasov and C. Vafa, On conformal field theories in four-dimensions, Nucl. Phys. B 533 (1998) 199 [hep-th/9803015] [INSPIRE].

[101] M. Bershadsky, Z. Kakushadze and C. Vafa, String expansion as large $N$ expansion of gauge theories, Nucl. Phys. B 523 (1998) 59 [hep-th/9803076] [InSPIRE].

[102] M. Bershadsky and A. Johansen, Large N limit of orbifold field theories, Nucl. Phys. B $\mathbf{5 3 6}$ (1998) 141 [hep-th/9803249] [INSPIRE].

[103] M. Schmaltz, Duality of nonsupersymmetric large $N$ gauge theories, Phys. Rev. D 59 (1999) 105018 [hep-th/9805218] [INSPIRE].

[104] J. Erlich and A. Naqvi, Nonperturbative tests of the parent/orbifold correspondence in supersymmetric gauge theories, JHEP 12 (2002) 047 [hep-th/9808026] [INSPIRE].

[105] E. Silverstein and Y.S. Song, On the critical behavior of D1-brane theories, JHEP 03 (2000) 029 [hep-th/9912244] [INSPIRE].

[106] D. Tong, Comments on condensates in nonsupersymmetric orbifold field theories, JHEP 03 (2003) 022 [hep-th/0212235] [INSPIRE].

[107] G. 't Hooft, A Two-Dimensional Model for Mesons, Nucl. Phys. B 75 (1974) 461 [InSPIRE].

[108] R. Dempsey, I.R. Klebanov and S.S. Pufu, Exact Symmetries and Threshold States in Two-Dimensional Models for QCD, arXiv:2101.05432 [INSPIRE].

[109] D.V. Vassilevich, Heat kernel expansion: User's manual, Phys. Rept. 388 (2003) 279 [hep-th/0306138] [INSPIRE].

[110] D.J. Gross and F. Wilczek, Ultraviolet Behavior of Nonabelian Gauge Theories, Phys. Rev. Lett. 30 (1973) 1343 [INSPIRE]. 
[111] T.P. Cheng, E. Eichten and L.-F. Li, Higgs Phenomena in Asymptotically Free Gauge Theories, Phys. Rev. D 9 (1974) 2259 [InSPIRE].

[112] N. Yamatsu, Finite-Dimensional Lie Algebras and Their Representations for Unified Model Building, arXiv:1511.08771 [INSPIRE].

[113] Y. Frishman and J. Sonnenschein, QCD in two-dimensions screening, confinement and novel nonAbelian solutions, Nucl. Phys. B 496 (1997) 285 [hep-th/9701140] [INSPIRE].

[114] S. Weinberg, Effective field theories in the large N limit, Phys. Rev. D 56 (1997) 2303 [hep-th/9706042] [INSPIRE].

[115] M. Moshe and J. Zinn-Justin, Quantum field theory in the large $N$ limit: A review, Phys. Rept. 385 (2003) 69 [hep-th/0306133] [INSPIRE].

[116] E. Witten, Nonabelian Bosonization in Two-Dimensions, Commun. Math. Phys. 92 (1984) 455 [INSPIRE].

[117] R.C. Myers, Dielectric branes, JHEP 12 (1999) 022 [hep-th/9910053] [INSPIRE].

[118] S. Lanza, F. Marchesano, L. Martucci and I. Valenzuela, Swampland Conjectures for Strings and Membranes, JHEP 02 (2021) 006 [arXiv:2006.15154] [INSPIRE].

[119] S. Lanza, F. Marchesano, L. Martucci and I. Valenzuela, The EFT stringy viewpoint on large distances, JHEP 21 (2020) 197 [arXiv:2104.05726] [INSPIRE].

[120] M. Billó, B. Craps and F. Roose, Anomalous couplings of type 0 D-branes, in NATO Advanced Study Institute: TMR Summer School on Progress in String Theory and M-theory, (1999) [hep-th/9908030] [INSPIRE].

[121] M. Dine and N. Seiberg, Is the Superstring Weakly Coupled?, Phys. Lett. B 162 (1985) 299 [INSPIRE].

[122] R. Antonelli, I. Basile and E. Hatefi, On All-Order Higher-Point Dp- $\overline{D p}$ Effective Actions, JCAP 10 (2019) 041 [arXiv: 1903.07540] [inSPIRE].

[123] E. Hatefi and P. Sundell, All-Order Quartic Couplings in Highly Symmetric D-brane-Anti-D-brane Systems, JHEP 05 (2021) 245 [arXiv:2103.06302] [INSPIRE].

[124] K.A. Meissner and G. Veneziano, Symmetries of cosmological superstring vacua, Phys. Lett. B 267 (1991) 33 [INSPIRE].

[125] K.A. Meissner, Symmetries of higher order string gravity actions, Phys. Lett. B 392 (1997) 298 [hep-th/9610131] [INSPIRE].

[126] O. Hohm and B. Zwiebach, T-duality Constraints on Higher Derivatives Revisited, JHEP 04 (2016) 101 [arXiv: 1510.00005] [INSPIRE].

[127] O. Hohm and B. Zwiebach, Non-perturbative de Sitter vacua via $\alpha^{\prime}$ corrections, Int. J. Mod. Phys. D 28 (2019) 1943002 [arXiv:1905.06583] [INSPIRE].

[128] O. Hohm and B. Zwiebach, Duality invariant cosmology to all orders in $\alpha$ ', Phys. Rev. D 100 (2019) 126011 [arXiv: 1905.06963] [inSPIRE].

[129] C. Krishnan, de Sitter, $\alpha^{\prime}$-Corrections \& Duality Invariant Cosmology, JCAP 10 (2019) 009 [arXiv: 1906.09257] [INSPIRE].

[130] I. Basile and A. Platania, Cosmological $\alpha^{\prime}$-corrections from the functional renormalization group, JHEP 21 (2021) 045 [arXiv:2101.02226] [inSPIRE]. 
[131] I. Basile and A. Platania, String Tension between de Sitter vacua and Curvature Corrections, arXiv:2103.06276 [INSPIRE].

[132] H. Bernardo, P.-R. Chouha and G. Franzmann, Kalb-Ramond backgrounds in $\alpha$ '-complete cosmology, JHEP 09 (2021) 109 [arXiv:2104.15131] [INSPIRE].

[133] J. Quintin, H. Bernardo and G. Franzmann, Cosmology at the top of the $\alpha$ ' tower, JHEP 07 (2021) 149 [arXiv: 2105.01083] [INSPIRE].

[134] H. Ooguri and C. Vafa, On the Geometry of the String Landscape and the Swampland, Nucl. Phys. B 766 (2007) 21 [hep-th/0605264] [INSPIRE].

[135] S.-J. Lee, W. Lerche and T. Weigand, Emergent Strings, Duality and Weak Coupling Limits for Two-Form Fields, arXiv: 1904.06344 [INSPIRE].

[136] S.-J. Lee, W. Lerche and T. Weigand, Emergent Strings from Infinite Distance Limits, arXiv: 1910.01135 [INSPIRE].

[137] F. Baume and J. Calderón Infante, Tackling the SDC in AdS with CFTs, JHEP 08 (2021) 057 [arXiv: 2011.03583] [INSPIRE].

[138] E. Perlmutter, L. Rastelli, C. Vafa and I. Valenzuela, A CFT Distance Conjecture, arXiv:2011.10040 [INSPIRE]. 